\title{
Topological properties of spaces of projective unitary representations
}

\author{
Jesús Espinoza ${ }^{1}$, Bernardo Uribe ${ }^{2, *}$ \\ ${ }^{1}$ Licenciatura en Matemáticas Aplicadas, Universidad del Papaloapan, Av. Ferrocarril s/n. 68400 \\ Ciudad Universitaria. Campus Loma Bonita, Oaxaca, México \\ ${ }^{2}$ Departamento de Matemáticas y Estadística, Universidad del Norte, Km 5 Vía Puerto Colombia, Barranquilla, Colombia
}

\begin{abstract}
Let $G$ be a compact and connected Lie group and $P U(\mathcal{H})$ be the group of projective unitary operators on an infinite dimensional separable Hilbert space $\mathcal{H}$ endowed with the strong operator topology. We study the space $\operatorname{hom}_{\mathrm{st}}(G, P U(\mathcal{H}))$ of continuous homomorphisms from $G$ to $P U(\mathcal{H})$ which are stable, namely the homomorphisms whose induced representation contains each irreducible representation an infinitely number of times. We show that the connected components of $\operatorname{hom}_{\mathrm{st}}(G, P U(\mathcal{H}))$ are parametrized by the isomorphism classes of $S^{1}$-central extensions of $G$, and that each connected component has the group $\operatorname{hom}\left(G, S^{1}\right)$ for fundamental group and trivial higher homotopy groups. We study the conjugation map $P U(\mathcal{H}) \rightarrow \operatorname{hom}_{\text {st }}(G, P U(\mathcal{H})), F \mapsto F \alpha F^{-1}$, we show that it has no local cross sections and we prove that for a map $B \rightarrow \operatorname{hom}_{\text {st }}(G, P U(\mathcal{H}))$ with $B$ paracompact of finite paracompact dimension, local lifts to $P U(\mathcal{H})$ do exist. (C) Acad. Colomb. Cienc. Ex. Fis. Nat. 2016.
\end{abstract}

Key words: Unitary Representation, Projective Unitary Representation.

Propiedades topológicas del espacio de representaciones unitarias proyectivas.

\section{Resumen}

Sea $G$ un grupo de Lie compacto y conexo y $P U(\mathcal{H})$ el grupo de operadores proyectivos e unitarios en un espacio de Hilbert separable e infinito dimensional $\mathcal{H}$, provisto de la topología fuerte de operadores. Estudiamos el espacio hom $\operatorname{st}_{\mathrm{s}}(\mathrm{G}, P U(\mathcal{H}))$ de homomorfismos continuos desde $G$ a $P U(\mathcal{H})$ que son estables, es decir homomorfismos cuyas representaciones inducidas contienen cada representación irreducible un número infinito

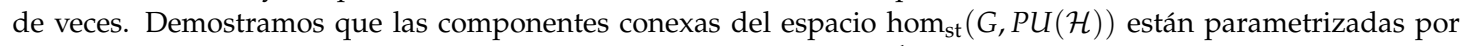
las clases de isomorfía de extensiones centrales de $G$ por el grupo $S^{1}$, y que cada componente conexa tiene por grupo fundamental al grupo $\operatorname{hom}\left(G, S^{1}\right)$ y sus grupos de homotopía superiores son triviales. Estudiamos la aplicación conjugación $P U(\mathcal{H}) \rightarrow \operatorname{hom}_{\mathrm{st}}(G, P U(\mathcal{H})), F \mapsto F \alpha F^{-1}$, demostramos que no tiene secciones locales y demostramos que para cualquier aplicación continua $B \rightarrow \operatorname{hom}_{\mathrm{st}}(G, P U(\mathcal{H}))$ con $B$ paracompacto de dimensión paracompacta finita, los levantamientos locales a $P U(\mathcal{H})$ sí existen. (C) Acad. Colomb. Cienc. Ex. Fis. Nat. 2016.

Palabras clave: Representación Unitaria, Representación Proyectiva Unitaria.

\section{Introduction}

The motivation to study the topological properties of the space $\operatorname{hom}_{\mathrm{st}}(G, P U(\mathcal{H}))$ of stable homomorphisms from a compact Lie group $G$ to the group of projective unitary operators on a Hilbert space $\mathcal{H}$ endowed with the topology of pointwise convergence, comes from realm of equivariant K-theory.

By a theorem of Atiyah and Jänich Jänich (1965) the Ktheory groups of a topological space $X$ may be obtained as the homotopy groups of the space

$$
\operatorname{map}(X, \operatorname{Fred}(\mathcal{H}))
$$

of continuous maps from $X$ to the $\operatorname{space} \operatorname{Fred}(\mathcal{H})$ of Fredholm operators on $\mathcal{H}$. Given any projective unitary bundle over $X$, namely a $P U(\mathcal{H})$-principal bundle $P U(\mathcal{H}) \rightarrow P \rightarrow X$, we may define a twisted version of the K-theory groups by taking the homotopy groups of the space of sections of the associated $\operatorname{Fred}(\mathcal{H})$ bundle

$$
P \times_{P U(\mathcal{H})} \operatorname{Fred}(\mathcal{H}) \rightarrow X .
$$

\footnotetext{
*Corresponding author:

Bernardo Uribe, bjongbloed@uninorte.edu.co

Received: November 11, 2015

Accepted: June 7, 2016
} 
These groups are called the twisted K-theory groups and they define a parametrized cohomology theory in the sense of May and Sigurdsson (2006) whenever we consider the category of pairs $(X, f)$ with $X$ a topological space and $f: X \rightarrow B P U(\mathcal{H})$ a map which recovers the $\operatorname{PU}(\mathcal{H})$ bundle $P$.

In the equivariant setup, namely when we consider the category of spaces with $G$ actions, the definition of the twisted equivariant K-theory is more intricate. We need to consider $G$ equivariant projective unitary stable bundles, namely $G$ equivariant $P U(\mathcal{H})$-principal bundles $P \rightarrow X$, such that the induced local homomorphism $G_{x} \rightarrow P U(\mathcal{H})$ is stable for $G_{x}$ the isotropy group of any $x \in X$, in order to define the twisted equivariant K-theory groups as the homotopy groups of the $G$ invariant sections of the associated bundle

$$
P \times_{P U(\mathcal{H})} \operatorname{Fred}(\mathcal{H}) \rightarrow X
$$

To prove that the twisted equivariant K-theory is a parametrized cohomology theory in the sense of May (1996) we would need to construct a universal $G$ equivariant projective unitary stable bundle as it was done in the non-equivariant case. The construction of this universal space can be done using classifying spaces of families of subgroups as it was done in Lück and Uribe (2014), though the property of being locally trivial depends on the existence of cross local sections on the conjugation map

$$
\begin{aligned}
P U(\mathcal{H}) & \rightarrow \operatorname{hom}_{\mathrm{st}}(G, P U(\mathcal{H})) \\
F & \mapsto F \alpha F^{-1} .
\end{aligned}
$$

Unfortunately such local cross sections fail to exist in general, as we shown in Theorem 4.8, and therefore the universal space that we can construct using families of subgroups fails to be locally trivial. Nevertheless when we restrict ourselves to consider only maps

$$
B \rightarrow \operatorname{hom}_{\mathrm{st}}(G, \operatorname{PU}(\mathcal{H}))
$$

with $B$ paracompact, we prove in Theorem 5.6 that these maps have indeed local lifts to $\operatorname{PU}(\mathcal{H})$. The previous result would imply that the universal space constructed using classifying spaces of families of subgroups done in Lück and Uribe (2014) would become a universal G equivariant projective unitary stable bundle for paracompact spaces, and hence, when restricted to paracompact spaces, the twisted equivariant K-theory would be a parametrized equivariant cohomology theory. We have not proven this last statement, but we believe it is true.
Besides the application of our results to K-theory, we also show the following facts. We study the space of stable unitary representations hom st $(G, U(\mathcal{H}))$ on a Hilbert space $\mathcal{H}$ and we generalize results of Dixmier-Douady on the infinite grassmannian $\operatorname{Gr}(\mathcal{H})$ to the space of unitary representations. We show in Corollary 4.10 that hom st $(G, U(\mathcal{H}))_{\mathcal{C}}$ is weakly homotopy equivalent to a point for any choice of irreducible representations $\mathcal{C} \subset \operatorname{Irrep}(G)$, we show that the space hom $\operatorname{st}_{\text {st }}(G U(\mathcal{H}))$ has as many connected components as $S^{1}$-central extensions of $G$ and that each connected component has hom $\left(G, S^{1}\right)$ for fundamental group and trivial higher homotopy groups.

The article is organized as follows. In Section 2 we recall the properties of the group of unitary operators endowed with the strong operator topology and we define the infinite grassmannian. In Section 3 we recall the definition of continuous field of Hilbert spaces done by DixmierDouady in Dixmier and Douady (1963) and we show the properties of the infinite grassmannian with respect to the existence of sections on the unitary group. In Section 4 we study the topological properties of the spaces

$$
\operatorname{hom}_{\text {st }}(G, U(\mathcal{H}))
$$

of stable continuous homomorphisms from a compact Lie group to the group of unitary operators. In Section 5 we study the topological properties of the space

$$
\operatorname{hom}_{\text {st }}(G, P U(\mathcal{H}))
$$

of stable continuous homomorphisms from a compact Lie group to the group of projective unitary operators. Finally, in Section 6 we show some applications to twisted equivariant K-theory of the results of the previous sections and we conclude with some ideas for further research.

Acknowledgements: The first author acknowledges the support of a CONACyT postdoctoral fellowship and of the Centro de Ciencias Matemáticas of the UNAM. The second author acknowledges the financial support of the Max Planck Institute for Mathematics in Bonn and of COLCIENCIAS through contract number FP44842-6172014.

\section{Spaces of operators and the infinite grassmannian}

Let $\mathcal{H}$ be a separable and infinite dimensional complex Hilbert space and denote by $\mathcal{B}(\mathcal{H})$ the vector space of bounded linear operators. The inner product $\langle$,$\rangle on \mathcal{H}$ induces the norm $|x|:=\sqrt{\langle x, x\rangle}$ for $x \in \mathcal{H}$, and we have 
the standard norm on the space of bounded linear operators

$$
|T|=\sup _{|x| \leq 1}|T x|
$$

The space $\mathcal{B}(\mathcal{H})$ can be endowed with several topologies, among them we have the strong operator topology and the compact-open topology. These topologies are of interest when studying principal bundles and therefore are the ones of interest in this paper.

Recall that in the strong operator topology, a subbasic open set is given by

$$
V(T, \varepsilon ; x)=\{S \in \mathcal{B}(\mathcal{H})|| S x-T x \mid<\varepsilon\}
$$

for any $T \in \mathcal{B}(\mathcal{H}), x \in \mathcal{H}$ and $\varepsilon>0$. In this topology, a sequence of bounded operators $\left\{T_{n}\right\}$ converges to $T \in \mathcal{B}(\mathcal{H})$ if and only if $T_{n} x \rightarrow T x$ for all $x \in \mathcal{H}$. On the other hand, a subbase for the compact-open topology on $\mathcal{B}(\mathcal{H})$ is given by the family of sets

$$
V(K, A)=\{S \in \mathcal{B}(\mathcal{H}) \mid S(K) \subset A\},
$$

where $K \subset \mathcal{H}$ is a compact set and $A \subset \mathcal{H}$ is an open set. Note that in the compact-open topology a sequence of bounded operators $\left\{T_{n}\right\}$ converges to $T \in \mathcal{B}(\mathcal{H})$ if and only if $\left.\left.T_{n}\right|_{K} \rightarrow T\right|_{K}$ uniformly for every compact set $K \subset \mathcal{H}$.

Neither of the previous topologies on $\mathcal{B}(\mathcal{H})$ are equivalent. However, if we restrict to the group of unitary operators on $\mathcal{H}$,

$$
U(\mathcal{H})=\left\{U \in \mathcal{B}(\mathcal{H}) \mid U U^{*}=U^{*} U=\operatorname{Id}_{\mathcal{H}}\right\},
$$

then we know that the strong operator topology and the compact-open topology agree on the group $U(\mathcal{H})$. Moreover, the group $U(\mathcal{H})$ endowed with any of these topologies is a Polish group, i.e. a completely metrizable topological group (see Espinoza and Uribe (2014)), and furthermore contractible (Dixmier and Douady, 1963, §11, Lem. 3). Let us recall the metric on $U(\mathcal{H})$ which recovers the strong operator topology since it will be needed in what follows.

Let $\left\{e_{j}\right\}_{j \in \mathbb{N}}$ be an orthonormal base of $\mathcal{H}$ and consider the embedding $\Psi: U(\mathcal{H}) \rightarrow \mathcal{H}^{\mathbb{N}}$ with $\Psi(T)=\left(T e_{j}\right)_{j \in \mathbb{N}}$. Any metric on $\mathcal{H}^{\mathbb{N}}$ which induces the product topology induces also a metric on $U(\mathcal{H})$ compatible with the strong operator topology. Therefore for any pairs of operators $T, U \in U(\mathcal{H})$ we may define their distance by the equation

$$
\langle T, U\rangle:=\sup _{n \in \mathbb{N}}\left\{\frac{\min \left\{\left|T e_{n}-U e_{n}\right|, 2\right\}}{n}\right\} .
$$

Note that with this metric $S^{1}$ acts by isometries on $U(\mathcal{H})$, i.e.

$$
\left\langle e^{i t} T, e^{i t} U\right\rangle=\langle T, U\rangle
$$

and moreover we have that

$$
\left\langle e^{i t} T, e^{i s} T\right\rangle=\left|e^{i t}-e^{i s}\right| .
$$

From now and on we will assume that $U(\mathcal{H})$ is endowed with the strong operator topology and with the metric defined above. The first consequence of this choice is the following lemma.

Lemma 2.1. Let $X$ be a topological space and let us consider $\mathcal{U}(\mathcal{H})$ with the strong operator topology. Then a map $\Psi: X \rightarrow U(\mathcal{H})$ is continuous if and only if the map

$$
\begin{aligned}
\psi: X \times \mathcal{H} & \longrightarrow \mathcal{H} \\
(x, h) & \longmapsto \Psi(x) h
\end{aligned}
$$

is continuous.

Proof. Consider $\left(x_{0}, h_{0}\right) \in X \times \mathcal{H}$ and let

$$
B\left(\Psi\left(x_{0}\right) h_{0}, \varepsilon\right)
$$

be an open ball in $\mathcal{H}$ with center at $\psi\left(x_{0}, h_{0}\right)=\Psi\left(x_{0}\right) h_{0}$ and radius $\varepsilon>0$. We will show that there exists an open set $A \subset X \times \mathcal{H}$ such that $\psi(A) \subset B\left(\Psi\left(x_{0}\right) h_{0}, \varepsilon\right)$.

Define $V\left(\Psi\left(x_{0}\right), \varepsilon / 2 ; h_{0}\right)=\left\{T \in U(\mathcal{H}) \mid T h_{0} \in\right.$ $\left.B\left(\Psi\left(x_{0}\right) h_{0}, \varepsilon / 2\right)\right\}$ which is an open set in the strong operator topology on $U(\mathcal{H})$. Then $U=\Psi^{-1}\left(V\left(\Psi\left(x_{0}\right), \varepsilon / 2 ; h_{0}\right)\right)$ is an open set in $X$.

Let us see that the open set

$$
A=U \times B\left(h_{0} ; \varepsilon / 2\right) \subset X \times \mathcal{H}
$$

has the desired properties. Indeed, for $(x, h) \in A$ we have

$$
\begin{aligned}
& \left|\psi(x, h)-\psi\left(x_{0}, h_{0}\right)\right| \\
& \quad=\left|\Psi(x) h-\Psi\left(x_{0}\right) h_{0}\right| \\
& \quad \leq\left|\Psi(x) h-\Psi(x) h_{0}\right|+\left|\Psi(x) h_{0}-\Psi\left(x_{0}\right) h_{0}\right| \\
& \quad=\left|h-h_{0}\right|+\left|\Psi(x) h_{0}-\Psi\left(x_{0}\right) h_{0}\right|<\frac{\varepsilon}{2}+\frac{\varepsilon}{2}
\end{aligned}
$$

and therefore $A \subset \psi^{-1}\left(B\left(\Psi\left(x_{0}\right) h_{0}, \varepsilon\right)\right)$.

By (Munkres, 2000, Thm. 46.11) the reciprocal statement is true whenever $U(\mathcal{H})$ is endowed with the compactopen topology. Since the strong operator topology and the compact-open topology agree on $U(\mathcal{H})$ the lemma follows. 
Recall that an orthogonal projector $P$ on the Hilbert space consist of an operator $P: \mathcal{H} \rightarrow \mathcal{H}$ such that $P^{2}=P$ with the property that $\operatorname{ker}(P)$ and $\operatorname{Im}(P)$ are orthogonal. Define the infinite grassmannian as follows

$$
\begin{aligned}
\operatorname{Gr}(\mathcal{H}):=\{P \in \mathcal{B}(\mathcal{H}) \mid & P^{2}=P, \operatorname{ker}(P)=\operatorname{Im}(P)^{\perp}, \\
& \operatorname{dim}(\operatorname{ker}(P))=\operatorname{dim}(\operatorname{Im}(P))=\infty\}
\end{aligned}
$$

and endow it with the strong operator topology.

Since the map

$$
\operatorname{Gr}(\mathcal{H}) \rightarrow U(\mathcal{H}), \quad P \mapsto 2 P-1
$$

is an embedding, and $U(\mathcal{H})$ is a Polish group, then $\operatorname{Gr}(\mathcal{H})$ is metrizable. Moreover, since $\operatorname{Gr}(\mathcal{H})$ is closed in $U(\mathcal{H})$ we have that the infinite grassmannian $\operatorname{Gr}(\mathcal{H})$ is a completely metrizable space.

\section{Continuous fields of Hilbert spaces}

The following is the definition of Dixmier and Douady Dixmier and Douady (1963) applied to the case of Hilbert spaces.

Consider $B$ a topological space and denote by $\mathcal{O}(B)$ the algebra of continuous complex valued functions on $B$. Let $(E(z))_{z \in B}$ be a family of Hilbert spaces. For $Y \subset B$, an element in $\prod_{z \in Y} E(z)$, i.e. an assignment $s$ defined on $Y$ such that $s(z) \in E(z)$ for all $z \in Y$, will be called a vector field over $Y$. For $s$ a vector field over $Y$, we will denote by $\|s\|$ the map $z \mapsto\|s(z)\|$ taking values in $\mathbb{R}$.

Definition 3.1. (Dixmier and Douady, 1963, Def. 1, pp 228) A continuous field of Hilbert spaces $\mathscr{E}$ over the topological space $B$ is a family $(E(z))_{z \in B}$ of Hilbert spaces, endowed with a set $\Gamma \subset \prod_{z \in B} E(z)$ of vector fields, such that:

- $\Gamma$ is a sub- $\mathcal{O}(B)$-module of $\prod_{z \in B} E(z)$.

- For all $z \in B$ and all $\xi \in E(z)$, there exists $s \in \Gamma$ such that $s(z)=\xi$.

- For all $s \in \Gamma$, the map $\|s\|$ is continuous.

- If $s \in \prod_{z \in B} E(z)$ is a vector field such that for all $z \in B$ and every $\varepsilon>0$ there exists $s^{\prime} \in \Gamma$ satisfying $\left\|s-s^{\prime}\right\| \leq \varepsilon$ on a neighborhood of $z$, then $s \in \Gamma$.

The elements of $\Gamma$ will be called continuous vector fields of $\mathscr{E}$.

Let $\mathcal{H}$ be a Hilbert space and $\Gamma$ the space of continuous maps from $B$ to $\mathcal{H}$. For every $z \in B$ define $E(z):=\mathcal{H}$. Then $\mathscr{E}=\left((E(z))_{z \in B}, \Gamma\right)$ is a continuous field of Hilbert spaces and will be called the constant field over $B$ defined by $\mathcal{H}$.

Following (Dixmier and Douady, 1963, §12), denote by $\mathscr{D}_{0}$ the constant field over $\operatorname{Gr}(\mathcal{H})$ defined by $\mathcal{H}$ and denote by $\mathscr{D}$ the canonical field over $\operatorname{Gr}(\mathcal{H})$ defined by the family of vector spaces $(P(\mathcal{H}))_{P \in \operatorname{Gr}(\mathcal{H})}$, where $\Gamma$ is the set of vector fields parametrized by the elements in $\mathcal{H}$

$$
\Gamma=\left\{\bar{\xi} \in \prod_{P \in \operatorname{Gr}(\mathcal{H})} P(\mathcal{H}) \mid \bar{\xi} \in \mathcal{H}\right\}
$$

with $\bar{\xi}(P):=P(\xi)$. Denote by $\mathscr{D}^{\perp}$ the family of vector spaces $\left(P(\mathcal{H})^{\perp}\right)_{P \in \operatorname{Gr}(\mathcal{H})}$ and note that both $\mathscr{D}$ and $\mathscr{D}^{\perp}$ are sub-fields of $\mathscr{D}_{0}$ and moreover $\mathscr{D} \oplus \mathscr{D}^{\perp} \cong \mathscr{D}_{0}$.

From Theorem 2 in (Dixmier and Douady, 1963, §12), it follows that the canonical field over the infinite grassmannian is trivial if $\operatorname{Gr}(\mathcal{H})$ has the norm topology. Nevertheless whenever $\operatorname{Gr}(\mathcal{H})$ is endowed with the strong operator topology, then the canonical field is not locally trivial (Dixmier and Douady, 1963, §16, Cor. 2).

The continuous field $\mathscr{D}$ over $\operatorname{Gr}(\mathcal{H})$ is a universal continuous field for continuous fields of infinite dimensional Hilbert spaces over paracompact spaces. This fact follows from (Dixmier and Douady, 1963, §14, Cor. 1) which we quote here: Let $\mathscr{E}$ be a continuous field of infinite dimensional and separable Hilbert spaces over the paracompact space $B$. Then there exist a continuous map $\phi: B \rightarrow \operatorname{Gr}(\mathcal{H})$ such that $\mathscr{E} \cong \phi^{*} \mathscr{D}$.

Dixmier and Douady show this fact in (Dixmier and Douady, 1963, §13, Thm. 3) by constructing vector fields $\left\{\bar{s}_{n}\right\}_{n \in \mathbb{N}} \subset \Gamma$ which are orthogonal over $\phi(B)$ and such that for all $P \in \phi(B)$ the set $\left\{\bar{s}_{n}(P)\right\}_{n \in \mathbb{N}}$ is an orthonormal base for $P(\mathcal{H})$. With these sections at hand Dixmier and Douady furthermore show in (Dixmier and Douady, $1963, \S 15$, Thm. 5) that $\mathscr{E}$ is trivializable.

Lemma 3.2. Let $B$ be a paracompact space of finite paracompact dimension and $\phi: B \rightarrow \operatorname{Gr}(\mathcal{H})$ a continuous map. Take $b_{0} \in B$ and define the conjugation map

$$
\begin{aligned}
\pi_{\phi\left(b_{0}\right)}: U(\mathcal{H}) & \longrightarrow \operatorname{Gr}(\mathcal{H}) \\
F & \longmapsto F \phi\left(b_{0}\right) F^{-1} .
\end{aligned}
$$

Then there exist a continuous map $\sigma: B \rightarrow U(\mathcal{H})$ such that the following diagram is commutative

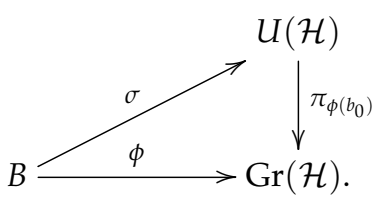


Proof. Since $\phi^{*} \mathscr{D} \oplus \phi^{*} \mathscr{D}^{\perp} \cong B \times \mathcal{H}$ and $\phi^{*} \mathscr{D}$ is trivializable, in (Dixmier and Douady, 1963, §14, Thm. 4) it is shown that there exist sections $\left\{\bar{s}_{n}\right\}_{n \in \mathbb{N}},\left\{\bar{t}_{n}\right\}_{n \in \mathbb{N}}$ for $\phi^{*} \mathscr{D}$ and $\phi^{*} \mathscr{D}^{\perp}$ respectively which are pointwise orthonormal, i.e. $\left\{\bar{s}_{n}(b)\right\}_{n \in \mathbb{N}}$ is an orthonormal base for $\phi(b)(\mathcal{H})$ and $\left\{\bar{t}_{n}(b)\right\}_{n \in \mathbb{N}}$ is an orthonormal base for $(\operatorname{Id}-\phi(b))(\mathcal{H})$.

Define the map $\psi: B \times \mathcal{H} \rightarrow \mathcal{H}$ by the assignment

$$
\psi\left(b, \bar{s}_{n}\left(b_{0}\right)\right)=\bar{s}_{n}(b) \text { and } \psi\left(b, \bar{t}_{n}\left(b_{0}\right)\right)=\bar{t}_{n}(b)
$$

and note that $\psi$ is continuous. By Lemma 2.1 the map $\sigma: B \rightarrow U(\mathcal{H})$ defined by the equation $\psi(b, h)=\sigma(b) h$ is continuous and we have that

$$
\sigma(b) \bar{s}_{n}\left(b_{0}\right)=\bar{s}_{n}(b) \text { and } \sigma(b) \bar{t}_{n}\left(b_{0}\right)=\bar{t}_{n}(b) .
$$

Therefore

$$
\begin{aligned}
\sigma(b) \phi\left(b_{0}\right)\left(\bar{s}_{n}\left(b_{0}\right)\right) & =\sigma(b)\left(\bar{s}_{n}\left(b_{0}\right)\right) \\
& =\bar{s}_{n}(b) \\
& =\phi(b)\left(\bar{s}_{n}(b)\right) \\
& =\phi(b) \sigma(b)\left(\bar{s}_{n}\left(b_{0}\right)\right)
\end{aligned}
$$

and $\sigma(b) \phi\left(b_{0}\right)\left(\bar{t}_{n}\left(b_{0}\right)\right)=0=\phi(b) \sigma(b)\left(\bar{t}_{n}\left(b_{0}\right)\right)$. Therefore $\sigma(b) \phi\left(b_{0}\right)=\phi(b) \sigma(b)$ and we have the desired equation $\sigma(b) \phi\left(b_{0}\right) \sigma(b)^{-1}=\phi(b)$.

We have just shown that over paracompact spaces we may construct sections of the conjugation map. But these sections fail to exist whenever the base is the infinite grassmannian.

Lemma 3.3. Let $Q \in \operatorname{Gr}(\mathcal{H})$ be a projector. Then, the map

$$
\begin{aligned}
\pi_{Q}: U(\mathcal{H}) & \longrightarrow \mathrm{Gr}(\mathcal{H}) \\
F & \longmapsto F Q F^{-1}
\end{aligned}
$$

has no global sections.

Proof. Let us proceed by contradiction. Suppose that there exists a continuous map $\sigma: \operatorname{Gr}(\mathcal{H}) \rightarrow U(\mathcal{H})$ such that $\sigma(P) Q \sigma(P)^{-1}=P$. By Lemma 2.1 we know that the evaluation map $(P, h) \mapsto \sigma(P) h$ is continuous. Hence the map $(P, h) \mapsto(P, \sigma(P) h)$ is also continuous, and its restriction

$$
\begin{aligned}
\operatorname{Gr}(\mathcal{H}) \times \operatorname{Im}(Q) & \longrightarrow \mathscr{D} \\
(P, h) & \longmapsto(P, \sigma(P) h)
\end{aligned}
$$

is continuous. This map trivializes the canonical field $\mathscr{D}$ over $\operatorname{Gr}(\mathcal{H})$ but this contradicts (Dixmier and Douady, $1963, \S 16$, Cor. 2) where it is shown that $\mathscr{D}$ is nowhere locally trivial.

The map $\pi_{Q}$ is surjective in $\operatorname{Gr}(\mathcal{H})$ since any two orthogonal projectors in $\operatorname{Gr}(\mathcal{H})$ are conjugate. Therefore the map
$\pi_{\mathrm{Q}}$ induces a continuous map

$$
U(\mathcal{H}) / U(\mathcal{H})_{Q} \longrightarrow \operatorname{Gr}(\mathcal{H}), \quad[F] \mapsto F Q F^{-1}
$$

where $U(\mathcal{H})_{Q}=\left\{T \in U(\mathcal{H}) \mid T Q T^{-1}=Q\right\}$ is the isotropy group of $Q$, which is moreover bijective but which is not a homeomorphism. This last statement follows from (Dixmier and Douady, 1963, §16, Cor. 2) where it is shown that $\mathscr{D}$ is nowhere locally trivial thus implying that the map $\pi_{Q}$ is not a $U(\mathcal{H})_{Q}$-principal bundle over $\operatorname{Gr}(\mathcal{H})$.

Nevertheless, the existence of extensions that were shown in Lemma 3.2 implies that the pullback $\phi^{*} U(\mathcal{H})$ over $B$ of a fixed map $\phi: B \rightarrow \operatorname{Gr}(\mathcal{H})$ is indeed a $U(\mathcal{H})_{\phi\left(b_{0}\right)^{-}}$ principal bundle. If $\phi^{*} U(\mathcal{H})=\{(b, F) \in B \times U(\mathcal{H})$ $\left.\phi(b)=\pi_{\phi\left(b_{0}\right)}(F)\right\}$ then the map

$$
B \times U(\mathcal{H})_{\phi\left(b_{0}\right)} \longrightarrow \phi^{*} U(\mathcal{H}), \quad(b, T) \mapsto(b, \sigma(b) T)
$$

is a $U(\mathcal{H})_{\phi\left(b_{0}\right)}$-bundle isomorphism.

We conclude this section by stating that $\operatorname{Gr}(\mathcal{H})$ is a classifying space for Hilbert space bundles over paracompact spaces. This follows from the following three facts: First, any continuous field of infinite dimensional Hilbert spaces over a paracompact space $B$ is isomorphic to the pullback over some map of the canonical field $\mathscr{D}$ over $\operatorname{Gr}(\mathcal{H})$. Second, any continuous field of infinite dimensional Hilbert spaces over a paracompact space is trivial. Third, the infinite grassmannian $\operatorname{Gr}(\mathcal{H})$ is contractible. Hence any two maps from $B$ to $\operatorname{Gr}(\mathcal{H})$ are homotopic, and any two continuous fields of infinite dimensional spaces over $B$ are isomorphic.

\section{Spaces of unitary representations}

Let $G$ be a compact Lie group and consider a continuous homomorphism $\alpha: G \rightarrow U(\mathcal{H})$. The homomorphism $\alpha$ induces the structure of a $G$ representation to $\mathcal{H}$ denoted by $\mathcal{H}_{\alpha}$ and we have a canonical decomposition of $\mathcal{H}_{\alpha}$ in isotypical components

$$
\mathcal{H} \cong \bigoplus_{V \in \operatorname{Irrep}(G)} \mathcal{H}_{\alpha}^{V}
$$

where $\operatorname{Irrep}(G)$ denotes the isomorphism classes of irreducible representations of $G, V$ is a representative of its isomorphism class of irreducible representation and $\mathcal{H}_{\alpha}^{V}$ is the isotypical subspace associated to $V$. By Schur's Lemma, the isotypical part associated to $V$ may be defined as the image of the evaluation map, i.e.

$$
\mathcal{H}_{\alpha}^{V}=\operatorname{Im}\left(e v: V \otimes \operatorname{hom}_{G}\left(V, \mathcal{H}_{\alpha}\right) \rightarrow \mathcal{H}_{\alpha}\right) .
$$

with $e v(v \otimes f)=f(v)$. 
Definition 4.1. Let $\alpha: G \rightarrow U(\mathcal{H})$ be a continuous homomorphism from a compact Lie group $G$ to $U(\mathcal{H})$. We say that the homomorphism is stable if all the isotypical components of $\mathcal{H}_{\alpha}$ are either infinite dimensional or zero dimensional. We will denote the set of stable homomorphisms as $\operatorname{hom}_{\text {st }}(G, U(\mathcal{H}))$.

The set of all homomorphisms hom $(G, U(\mathcal{H}))$ can be endowed with the subspace topology of the compact-open topology of the space

$$
\operatorname{map}(G, U(\mathcal{H}))
$$

of continuous maps from $G$ to $U(\mathcal{H})$, and a subbase for this topology is given by the family of sets

$$
\begin{aligned}
& \mathcal{V}(a, \varepsilon ; x):= \\
& \{\gamma \in \operatorname{hom}(G, U(\mathcal{H})) \mid\|\gamma(g)-a(g) x\|<\varepsilon, g \in G\}
\end{aligned}
$$

for $a \in \operatorname{hom}(G, U(\mathcal{H})), \varepsilon>0$ and $x \in \mathcal{H}$.

On the other hand, since $G$ is compact and $U(\mathcal{H})$ is metrizable, we may also endow

$$
\operatorname{map}(G, U(\mathcal{H}))
$$

with the supremum metric, i.e. for

$$
\alpha, \beta \in \operatorname{map}(G, U(\mathcal{H}))
$$

we define

$$
d_{\text {sup }}(\alpha, \beta)=\sup \{d(\alpha(g), \beta(g)) \mid g \in G\} .
$$

By (Munkres, 2000, Thm. 46.8) we know that these two topologies agree. Moreover, since $U(\mathcal{H})$ is complete we know that $\operatorname{map}(G, U(\mathcal{H}))$ is also complete (Munkres, 2000, Thm. 43.5).

Lemma 4.2. The space $\operatorname{hom}(G, U(\mathcal{H}))$ is a complete metric space.

Proof. Since $\operatorname{map}(G, U(\mathcal{H}))$ is complete we may take a convergent sequence $\left\{\alpha_{n}\right\}_{n \in \mathbb{N}}$ of homomorphisms which converge to $\alpha \in \operatorname{map}(G, U(\mathcal{H}))$. We know that

$$
\alpha_{n}(g) \alpha_{n}(h) \rightarrow \alpha(g) \alpha(h)
$$

and $\alpha_{n}(g h) \rightarrow \alpha(g h)$, and since $\alpha_{n}(g) \alpha_{n}(h)=\alpha_{n}(g h)$ we conclude that

$$
\alpha(g) \alpha(h)=\alpha(g h) .
$$

Therefore $\alpha$ is also a homomorphism and hence $\operatorname{hom}(G, U(\mathcal{H}))$ is complete.

Lemma 4.3. The space of stable homomorphisms $\operatorname{hom}_{\mathrm{st}}(G, U(\mathcal{H}))$ is not closed in $\operatorname{hom}(G, U(\mathcal{H}))$.

Proof. We will show a convergent sequence in $\operatorname{hom}_{\text {st }}\left(S^{1}, U(\mathcal{H})\right)$ whose limit is not stable. The argument for any compact Lie group is similar.
Let $\left\{e_{j}\right\}_{j \in \mathbb{N}}$ be an orthonormal base of $\mathcal{H}$. Define the homomorphisms $\alpha_{k}: S^{1} \rightarrow U(\mathcal{H})$ by the assignment

$$
\alpha_{k}\left(e^{i \theta}\right)\left(e_{2 j}\right)=e_{2 j}
$$

and

$$
\alpha_{k}\left(e^{i \theta}\right)\left(e_{2 j+1}\right)=\left\{\begin{array}{cll}
e_{2 j+1} & \text { if } \quad 0<j<k \\
e^{i \theta} e_{2 j+1} & \text { if } \quad j \geq k \text { or } j=0
\end{array}\right.
$$

and note that the $\alpha_{k}$ 's are stable. Since

$$
\lim _{k \rightarrow \infty} \alpha_{k}\left(e^{i \theta}\right) e_{j}=e_{j}
$$

for $j>1$ and

$$
\lim _{k \rightarrow \infty} \alpha_{k}\left(e^{i \theta}\right) e_{1}=e^{i \theta} e_{1}
$$

we know that $\lim _{k \rightarrow \infty} \alpha_{k}$ does not belong to the space of stable homomorphisms.

Lemma 4.4. The space $\operatorname{hom}_{\mathrm{st}}(G, U(\mathcal{H}))$ of stable homomorphisms is not open in $\operatorname{hom}(G, U(\mathcal{H}))$.

Proof. We will prove that for any basic open set $\mathcal{V} \subset$ $\operatorname{hom}(G, U(\mathcal{H}))$ and $a \in \mathcal{V}$ a stable homomorphism, there exist a non stable homomorphism $b \in \mathcal{V}$.

Fix $\varepsilon>0$ and $x_{1}, \ldots, x_{n} \in \mathcal{H}$. Define

$$
\begin{aligned}
\mathcal{V}=\mathcal{V}\left(a, \varepsilon ; x_{1}, \ldots, x_{n}\right) & \\
=\{\gamma \in \operatorname{hom}(G, U(\mathcal{H})) \mid & \left\|\gamma(g) x_{k}-a(g) x_{k}\right\|<\varepsilon, \\
& \forall g \in G, \forall k=1, \ldots, n\}
\end{aligned}
$$

and let $H=V_{1} \oplus \cdots \oplus V_{n}$ be the direct sum of the irreducible representations $V_{k}$ of $G$, such that $x_{k} \in V_{k}$ for each $k=1, \ldots, n$. It follows that $H$ is $a$-invariant and finite dimensional.

Let $b: G \rightarrow U(\mathcal{H})$ be given by

$$
b(g) x= \begin{cases}a(g) x & x \in H, \\ x & x \in \mathcal{H} \ominus H .\end{cases}
$$

Then $b \in \mathcal{V}$ by construction, but $a$ and $b$ are not unitary equivalent, i.e. $b$ is not stable.

Lemma 4.5. The space $\operatorname{hom}_{\mathrm{st}}(G, U(\mathcal{H}))$ of stable homomorphisms is dense in $\operatorname{hom}(G, U(\mathcal{H}))$.

Proof. Let $\alpha \in \operatorname{hom}(G, U(\mathcal{H}))$ be a homomorphism and let $\mathcal{V}\left(\alpha, \varepsilon ; x_{1}, \ldots, x_{n}\right)$ be a basic open set based at $\alpha \in \mathcal{V}$. Consider the finite dimensional and $\alpha$-invariant space $H=V_{1} \oplus \cdots \oplus V_{n}$ given by the direct sum of the irreducible representations $V_{k}$ of $G$, such that $x_{k} \in V_{k}$ for each $k=1, \ldots, n$. If $\psi$ is an isometric isomorphism $\psi: L^{2}(G) \otimes L^{2}([0,1]) \rightarrow \mathcal{H} \ominus H$, then

$$
b(g) x= \begin{cases}\alpha(g) x & x \in H \\ \psi \alpha(g) \psi^{-1} x & x \in \mathcal{H} \ominus H\end{cases}
$$


is a stable homomorphism and $b \in \mathcal{V}$.

Definition 4.6. Let $\mathcal{C} \subset \operatorname{Irrep}(G)$ be a choice of irreducible representations of the group $G$. Define

$$
\begin{aligned}
& \operatorname{hom}(G, U(\mathcal{H}))_{\mathcal{C}}:= \\
& \left\{\alpha \in \operatorname{hom}(G, \mathcal{U}(\mathcal{H})) \mid \operatorname{dim}\left(\mathcal{H}_{\alpha}^{V}\right)=0 \text { for } V \notin \mathcal{C}\right\}
\end{aligned}
$$

to be the space of homomorphisms which induce representations where only the irreducible representations in $\mathcal{C}$ appear. Define

$$
\begin{aligned}
& \operatorname{hom}_{\mathrm{st}}(G, U(\mathcal{H}))_{\mathcal{C}}:= \\
& \operatorname{hom}(G, U(\mathcal{H}))_{\mathcal{C}} \bigcap \operatorname{hom}_{\mathrm{st}}(G, U(\mathcal{H})) .
\end{aligned}
$$

We have therefore

$$
\begin{aligned}
& \operatorname{hom}_{\text {st }}(G, U(\mathcal{H}))_{\mathcal{C}}:= \\
& \left\{\alpha \in \operatorname{hom}(G, \mathcal{U}(\mathcal{H})) \mid \operatorname{dim}\left(\mathcal{H}_{\alpha}^{V}\right)=0 \text { for } V \notin \mathcal{C}\right. \\
& \text { and } \left.\operatorname{dim}\left(\mathcal{H}_{\alpha}^{V}\right)=\infty \text { for } V \in \mathcal{C}\right\} .
\end{aligned}
$$

The spaces $\operatorname{hom}_{\text {st }}(G, U(\mathcal{H}))_{\mathcal{C}}$ are neither closed nor open, nevertheless the action by conjugation of $U(\mathcal{H})$ on hom $_{\mathrm{st}}(G, U(\mathcal{H}))_{\mathcal{C}}$ is transitive and we are interested in studying the properties of this action.

Definition 4.7. Take a stable homomorphism $\alpha \in$ hom $_{\text {st }}(G, U(\mathcal{H}))_{\mathcal{C}}$. Define the conjugation map

$$
\pi_{\alpha}: U(\mathcal{H}) \rightarrow \operatorname{hom}_{\text {st }}(G, U(\mathcal{H}))_{\mathcal{C}}, \quad F \mapsto F \alpha F^{-1} .
$$

The map $\pi_{\alpha}$ is continuous and is surjective. Any other stable homomorphism

$$
\alpha^{\prime} \in \operatorname{hom}_{\text {st }}(G, U(\mathcal{H}))_{\mathcal{C}}
$$

induces an isomorphism $\mathcal{H} \cong \bigoplus_{V \in \mathcal{C}} \mathcal{H}_{\alpha^{\prime}}^{V}$. For each $V \in \mathcal{C}$ choose a G-equivariant unitary isomorphism $F^{V}: \mathcal{H}_{\alpha}^{V} \cong$ $\mathcal{H}_{\alpha^{\prime}}^{V}$ and assemble them into a G-equivariant unitary automorphism

$$
F:=\bigoplus_{V \in \mathcal{C}} F^{V}: \mathcal{H} \cong \mathcal{F} .
$$

By construction the unitary automorphism satisfies $F \alpha(g)=\alpha^{\prime}(g) F$ for all $g \in G$, and therefore $F \alpha F^{-1}=\alpha^{\prime}$.

The surjectivity of the map $\pi_{\alpha}$ implies that the map

$$
\begin{aligned}
U(\mathcal{H}) / U(\mathcal{H})_{\alpha} & \longrightarrow \operatorname{hom}_{\mathrm{st}}(G, U(\mathcal{H}))_{\mathcal{C}} \\
{[F] } & \mapsto F \alpha F^{-1}
\end{aligned}
$$

is a continuous map which is moreover bijective, where $U(\mathcal{H})_{\alpha}:=\left\{T \in U(\mathcal{H}) \mid T \alpha T^{-1}=\alpha\right\}$ is the isotropy group of $\alpha$. We will show that this map is not a homeomorphism, thus implying that the $\pi_{\alpha}$ is not a $U(\mathcal{H})_{\alpha}$-principal bundle. Nevertheless, the pullback of $\pi_{\alpha}$ for maps defined on paracompact spaces is indeed a $U(\mathcal{H})_{\alpha}$-principal bundle.
Theorem 4.8. Suppose that $\mathcal{C}$ contains the trivial representation, then the conjugation map $\pi_{\alpha}: U(\mathcal{H}) \rightarrow$ $\operatorname{hom}_{\mathrm{st}}(G, U(\mathcal{H}))_{\mathcal{C}}$ has no sections.

Proof. Note that in the case that $G=\mathbb{Z} / 2 \mathbb{Z}$ we have a homeomorphism

$$
\operatorname{hom}_{\text {st }}(\mathbb{Z} / 2 \mathbb{Z}, U(\mathcal{H})) \stackrel{\cong}{G} \mathrm{Gr}(\mathcal{H})
$$

where the homomorphism

$$
\beta \in \operatorname{hom}_{\text {st }}(\mathbb{Z} / 2 \mathbb{Z}, U(\mathcal{H}))
$$

is mapped to the orthogonal projector

$$
\frac{1}{2}(\beta(1)+\beta(-1)) \text {. }
$$

The same proof of Lemma 3.3 shows that the map $\pi_{\alpha}$ : $U(\mathcal{H}) \rightarrow \operatorname{hom}_{\text {st }}(\mathbb{Z} / 2 \mathbb{Z}, U(\mathcal{H}))$ has no sections.

The proof of the general case is based on the non existence of sections for the canonical field over the infinite grassmannian. We just need to find an appropriate injective map from $\operatorname{Gr}(\mathcal{H})$ to $\operatorname{hom}_{\mathrm{st}}(G, U(\mathcal{H}))_{\mathcal{C}}$.

Choose a representation $V \in \mathcal{C}$ different from the trivial representation. Take the isotypical decomposition of $\mathcal{H} \cong \bigoplus_{W \in \mathcal{C}} \mathcal{H}_{\alpha}^{W}$ defined by $\alpha$. Consider the infinite grassmannian

$$
\operatorname{Gr}\left(\operatorname{hom}_{G}\left(V, \mathcal{H}_{\alpha}^{V}\right)\right)
$$

of the Hilbert space $\operatorname{hom}_{G}\left(V, \mathcal{H}_{\alpha}^{V}\right)$ and for each $Z \in$ $\operatorname{Gr}\left(\operatorname{hom}_{G}\left(V, \mathcal{H}_{\alpha}^{V}\right)\right)$ denote by $e v(V \otimes Z)$ the subspace of $\mathcal{H}_{\alpha}^{V}$ defined by the image of $V \otimes Z$ under the evaluation map

$$
\text { ev }: V \otimes \operatorname{hom}_{G}\left(V, \mathcal{H}_{\alpha}^{V}\right) \rightarrow \mathcal{H}_{\alpha}^{V}
$$

with $e v(v \otimes f)=f(v)$ that was previously defined in (2).

Denote by $e v(V \otimes Z)^{\perp_{\mathcal{H}_{\alpha}^{V}}}$ the orthogonal complement of $e v(V \otimes Z)$ in $\mathcal{H}_{\alpha}^{V}$, i.e

$$
e v(V \otimes Z) \oplus e v(V \otimes Z)^{\perp_{\mathcal{H}}^{V}} \cong \mathcal{H}_{\alpha}^{V}
$$

and define the map

$$
\begin{aligned}
\Phi: \operatorname{Gr}\left(\operatorname{hom}_{G}\left(V, \mathcal{H}_{\alpha}^{V}\right)\right) & \rightarrow \operatorname{hom}_{\text {st }}(G, U(\mathcal{H}))_{\mathcal{C}} \\
Z & \mapsto \Phi(Z)
\end{aligned}
$$

with

$$
\Phi(Z)(g)\left(v \oplus v^{\prime}\right):=\alpha(g) v \oplus v^{\prime}
$$

where

$$
v \in\left(e v(V \otimes Z) \oplus \bigoplus_{W \in \mathcal{C}, W \neq V} \mathcal{H}_{\alpha}^{V}\right)
$$

$v^{\prime} \in e v(V \otimes Z)^{\perp_{\mathcal{H}_{\alpha}^{V}}}$ and $g \in G$. 
Note that the homomorphism $\Phi(Z)$ only disagrees with $\alpha$ on the subspace $e v(V \otimes Z)^{\perp_{\mathcal{H}_{\alpha}^{V}}}$, that the isotypical subspace of the homomorphism $\Phi(Z)$ associated to $V$ is precisely $e v(V \otimes Z)$, i.e.

$$
\mathcal{H}_{\Phi(Z)}^{V}=e v(V \otimes Z)
$$

and that

$$
\operatorname{hom}_{G}\left(V, \mathcal{H}_{\Phi(Z)}^{V}\right)=Z
$$

The map $\Phi$ is continuous since it can be defined as the composition of projections, and it is moreover injective.

Choose a base point $Z_{0} \in \operatorname{Gr}\left(\operatorname{hom}_{G}\left(V, \mathcal{H}_{\alpha}^{V}\right)\right)$ and

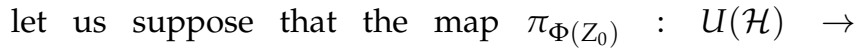
$\operatorname{hom}_{\text {st }}(G, U(\mathcal{H}))_{\mathcal{C}}, F \mapsto F \Phi\left(Z_{0}\right) F^{-1}$ has a section $\sigma$ : $\operatorname{hom}_{\text {st }}(G, U(\mathcal{H}))_{\mathcal{C}} \rightarrow U(\mathcal{H})$. Hence we would have that for all $g \in G$ the following equality holds

$$
\sigma(\Phi(Z))\left(\Phi\left(Z_{0}\right)(g)\right)=(\Phi(Z)(g)) \sigma(\Phi(Z)),
$$

which implies that $\sigma(\Phi(Z))$ induces a G-equivariant unitary isomorphism between the isotypical components

$$
\left.\sigma(\Phi(Z))\right|_{\mathcal{H}_{\Phi\left(Z_{0}\right)}^{W}:}: \mathcal{H}_{\Phi\left(Z_{0}\right)}^{W} \cong \mathcal{H}_{\Phi(Z)}^{W}
$$

and in particular it induces a G-equivariant isomorphism

$$
\left.\sigma(\Phi(Z))\right|_{e v\left(V \otimes Z_{0}\right)}: e v\left(V \otimes Z_{0}\right) \stackrel{\cong}{\rightarrow} e v(V \otimes Z) .
$$

Denote by $\mathscr{D}$ the canonical continuous field of Hilbert spaces over $\operatorname{Gr}\left(\operatorname{hom}_{G}\left(V, \mathcal{H}_{\alpha}^{V}\right)\right)$ defined as follows:

$$
\begin{aligned}
\mathscr{D} & :=\{(Z, f) \in \\
& \left.\operatorname{Gr}\left(\operatorname{hom}_{G}\left(V, \mathcal{H}_{\alpha}^{V}\right)\right) \times \operatorname{hom}_{G}\left(V, \mathcal{H}_{\alpha}^{V}\right) \mid f \in Z\right\},
\end{aligned}
$$

and construct the following map:

$$
\begin{aligned}
\theta: \operatorname{Gr}\left(\operatorname{hom}_{G}\left(V, \mathcal{H}_{\alpha}^{V}\right)\right) \times Z_{0} & \rightarrow \mathscr{D} \\
\left(Z, f_{0}\right) & \mapsto\left(Z, \sigma(\Phi(Z)) \circ f_{0}\right) .
\end{aligned}
$$

Note that the homomorphism $\sigma(\Phi(Z)) \circ f_{0}$ belongs to $Z$, since the image of $f$ lies in $\mathcal{H}_{\Phi\left(Z_{0}\right)}^{V}$ and the unitary isomorphism

$$
\left.\sigma(\Phi(Z))\right|_{\mathcal{H}_{\Phi\left(Z_{0}\right)}^{V}}: \mathcal{H}_{\Phi\left(Z_{0}\right)}^{V} \cong \mathcal{H}_{\Phi(Z)}^{V}
$$

is G-equivariant; hence

$$
\sigma(\Phi(Z)) \circ f_{0} \in \operatorname{hom}_{G}\left(V, \mathcal{H}_{\Phi(Z)}^{V}\right)=Z .
$$

Moreover the map $\theta$ is continuous since $\sigma$ is continuous, and its inverse map is simply $\theta^{-1}(Z, f)=$ $\left(Z, \sigma(\Phi(Z))^{-1} f\right)$. Therefore $\theta$ would be a trivialization of the canonical field $\mathscr{D}$ which contradicts (Dixmier and Douady, 1963, §16, Cor. 2) where it is shown that $\mathscr{D}$ is nowhere locally trivial.
Then the section $\sigma$ cannot exist and the theorem follows.

Theorem 4.9. Let $K$ be an abelian compact Lie group, B a paracompact space of finite paracompact dimension, with base point $b_{0} \in B$ and $f: B \rightarrow \operatorname{hom}_{\text {st }}(K, U(\mathcal{H}))_{\mathcal{C}}$ a continuous map with $\mathcal{C} \subset \operatorname{Irrep}(K)$. Then there exist an extension $\sigma: B \rightarrow U(\mathcal{H})$ that makes the following diagram commutative

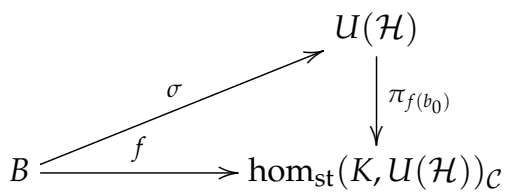

Proof. Since all irreducible representations of $K$ are 1dimensional, let us encode the information of each irreducible $V \in \operatorname{Irrep}(K)$ by a homomorphism $\chi_{V}: K \rightarrow$ $S^{1} \subset \mathbb{C}^{*}$. Choose a normalized left and right invariant measure on $K$, and for any $\alpha \in \operatorname{hom}_{\text {st }}(K, U(\mathcal{H}))_{\mathcal{C}}$ define the operator

$$
\psi_{V}(\alpha): \mathcal{H} \rightarrow \mathcal{H}, \quad h \mapsto \int_{K} \alpha(k) \chi_{V}\left(k^{-1}\right) h \mathrm{~d} k .
$$

Whenever $h \in \mathcal{H}_{\alpha}^{V}$ we have that $\psi_{V}(\alpha) h=h$, and whenever $h \in \mathcal{H}_{\alpha}^{W}$ for $W \neq V$ then $\psi_{V}(\alpha) h=0$. Therefore the operator $\psi_{V}(\alpha)$ is equivalent to the orthogonal projector $P_{\mathcal{H}_{\alpha}^{V}}$ that projects $\mathcal{H}$ to $\mathcal{H}_{\alpha}^{V}$. For $V \in \mathcal{C}$ the assignment $\psi_{V}$ defines a map

$$
\begin{aligned}
\psi_{V}: \operatorname{hom}_{\mathrm{st}}(K, U(\mathcal{H}))_{\mathcal{C}} & \rightarrow \operatorname{Gr}(\mathcal{H}) \\
\alpha & \mapsto \psi_{V}(\alpha)=P_{\mathcal{H}_{\alpha}^{V}}
\end{aligned}
$$

which is continuous since the integration is over a compact Lie group.

Consider the composition $\psi_{V} \circ f: B \rightarrow \operatorname{Gr}(\mathcal{H})$ and note that $\left(\psi_{V} \circ f\right)^{*} \mathscr{D}$ is trivializable. By (Dixmier and Douady, 1963, §14, Thm. 4) we know that there exist orthogonal sections $\left\{s_{n}^{V}\right\}_{n \in \mathbb{N}}$ of $\left(\psi_{V} \circ f\right)^{*} \mathscr{D}$ which satisfy $\bar{s}_{n}^{V}(b) \in$ $\mathcal{H}_{\psi_{V}(f(b))}^{V}$ and that moreover the vectors $\left\{s_{n}^{V}(b)\right\}_{n \in \mathbb{N}}$ are an orthogonal base of $\mathcal{H}_{\psi_{V}(f(b))}^{V}$.

Define the map $\phi: B \times \mathcal{H} \rightarrow \mathcal{H}$ by the assignment

$$
\phi\left(b, s_{n}^{V}\left(b_{0}\right)\right)=s_{n}^{V}(b)
$$

where $V$ runs over the irreducible representations in $\mathcal{C}$ and $\mathcal{H}$ is viewed as $\mathcal{H} \cong \bigoplus_{V \in \mathcal{C}} \mathcal{H}_{f\left(b_{0}\right)}^{V}$ in the source and as $\mathcal{H} \cong \bigoplus_{V \in \mathcal{C}} \mathcal{H}_{f(b)}^{V}$ in the target. Since the sections $s_{n}^{V}$ are continuous, the map $\phi$ is continuous.

By Lemma 2.1 the map $\sigma: B \rightarrow U(\mathcal{H})$ defined by the equation $\sigma(b) h:=\phi(b, h)$ is continuous and we have that

$$
\sigma(b) s_{n}^{V}\left(b_{0}\right)=s_{n}^{V}(b)
$$


for all $V \in \mathcal{C}$. In particular we have that

$$
\sigma(b) P_{\mathcal{H}_{f\left(b_{0}\right)}^{V}} \sigma(b)^{-1}=P_{\mathcal{H}_{f(b)}^{V}}
$$

and therefore

$$
\sigma(b) \psi_{V}\left(f\left(b_{0}\right)\right) \sigma(b)^{-1}=\psi_{V}(f(b))
$$

for all $V \in \mathcal{C}$.

Define the map

$$
\begin{aligned}
\Psi: \operatorname{hom}_{\mathrm{st}}(K, \mathcal{U}(\mathcal{H}))_{\mathcal{C}} & \rightarrow \prod_{V \in \mathcal{C}} \operatorname{Gr}(\mathcal{H}) \\
\alpha & \mapsto \prod_{V \in \mathcal{C}} \psi_{V}(\alpha)
\end{aligned}
$$

and note that the argument above implies that $\sigma$ makes the following diagram commutative

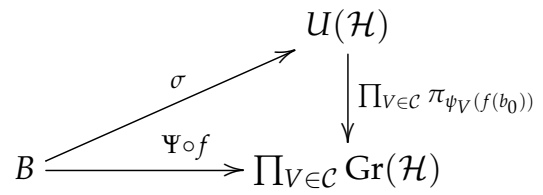

where $\pi_{\psi_{V}\left(f\left(b_{0}\right)\right)}: \mathcal{U}(\mathcal{H}) \rightarrow \operatorname{Gr}(\mathcal{H})$ is the conjugation map $\pi_{\psi_{V}\left(f\left(b_{0}\right)\right)}(F)=F \psi_{V}\left(f\left(b_{0}\right)\right) F^{-1}$.

Finally note that the map

$$
\Psi: \operatorname{hom}_{\mathrm{st}}(K, U(\mathcal{H}))_{\mathcal{C}} \rightarrow \prod_{V \in \mathcal{C}} \operatorname{Gr}(\mathcal{H})
$$

is injective since for abelian groups the isotypical spaces determine the homomorphism. Therefore the following diagram is commutative

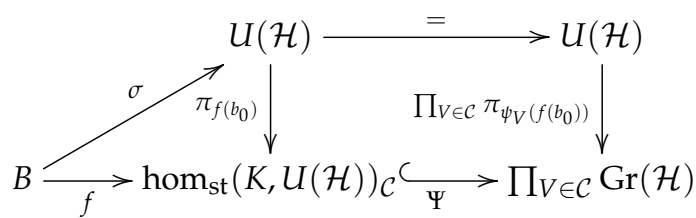

and the theorem follows.

Corollary 4.10. Let $G$ be a compact and connected Lie group, $B$ a paracompact space with finite paracompact dimension, with base point $b_{0} \in B$ and $f: B \rightarrow \operatorname{hom}_{\text {st }}(G, U(\mathcal{H}))_{\mathcal{C}}$ a continuous map with $\mathcal{C} \subset \operatorname{Irrep}(G)$. Then there exist an extension $\sigma: B \rightarrow U(\mathcal{H})$ that makes the following diagram commutative

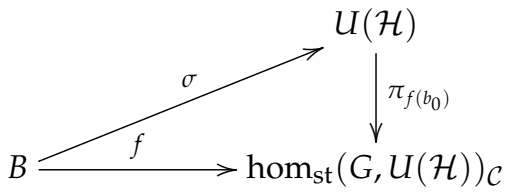

Proof. Let $K$ be a maximal abelian subgroup of $G$ and denote by $\iota: K \rightarrow G$ the inclusion. Recall that any representation of the group $G$ is uniquely determined by its restriction to a maximal abelian subgroup, and therefore the restriction map

$$
\iota^{*}: \operatorname{hom}_{\mathrm{st}}(G, \mathcal{U}(\mathcal{H}))_{\mathcal{C}} \hookrightarrow \operatorname{hom}_{\mathrm{st}}(K, \mathcal{U}(\mathcal{H}))_{\iota^{*} \mathcal{C}}
$$

is injective. Here we have denoted by $\iota^{*} \mathcal{C} \subset \operatorname{Irrep}(K)$ the set of irreducible representations in $K$ which appear as restrictions of representations $V$ of $\mathcal{C}$.

By Theorem 4.9 we know that there exists $\sigma$ for the composition map

$$
\iota^{*} \circ f: B \rightarrow \operatorname{hom}_{\mathrm{st}}(K, U(\mathcal{H}))_{\iota^{*} \mathcal{C}},
$$

and since $\iota^{*}$ is injective, the following diagram commutes

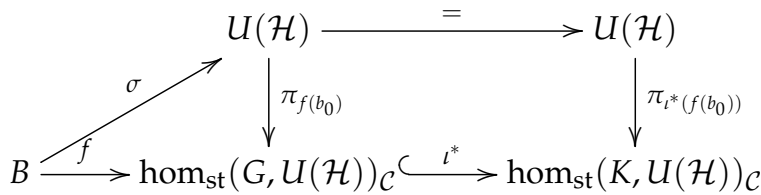

and the corollary follows.

The previous results have the following consequence:

Theorem 4.11. Let $G$ be a compact Lie group which moreover is connected or abelian, and $\mathcal{C} \subset \operatorname{Irrep}(G)$ a choice of irreducible representations. Then the space $\operatorname{hom}_{\mathrm{st}}(G, U(\mathcal{H}))_{\mathcal{C}}$ is weakly homotopy equivalent to a point.

Proof. We know that $\operatorname{hom}_{\mathrm{st}}(G, U(\mathcal{H}))_{\mathcal{C}}$ is connected since the conjugation map

$$
\begin{aligned}
\pi_{\alpha}: U(\mathcal{H}) & \rightarrow \operatorname{hom}_{\mathrm{st}}(G, U(\mathcal{H}))_{\mathcal{C}} \\
F & \mapsto F \alpha F^{-1}
\end{aligned}
$$

is surjective for any choice of $\alpha \in \operatorname{hom}_{\text {st }}(G, U(\mathcal{H}))_{\mathcal{C}}$.

Take any base point map

$$
f:\left(S^{n}, *\right) \rightarrow\left(\operatorname{hom}_{\text {st }}(G, U(\mathcal{H}))_{\mathcal{C}}, \alpha\right)
$$

and note that by Theorem 4.9 and Corollary 4.10 there exists $\sigma:\left(S^{n}, *\right) \rightarrow(U(\mathcal{H}), \mathrm{Id})$ such that $f=\pi_{\alpha} \circ \sigma$. Since $U(\mathcal{H})$ is contractible by (Dixmier and Douady, 1963, §11, Lem. 3), there exists $\widetilde{\sigma}:\left(B^{n+1}, *\right) \rightarrow(U(\mathcal{H}), I d)$ such that $\left.\widetilde{\sigma}\right|_{S^{n}}=\sigma$. Hence we have that

$$
\pi_{\alpha} \circ \widetilde{\sigma}:\left(B^{n+1}, *\right) \rightarrow\left(\operatorname{hom}_{\mathrm{st}}(G, U(\mathcal{H}))_{\mathcal{C}}, \alpha\right)
$$

satisfies $\left.\pi_{\alpha} \circ \tilde{\sigma}\right|_{S^{n}}=f$ and therefore the homotopy groups of hom st $(G, U(\mathcal{H}))_{\mathcal{C}}$ are all trivial. 


\section{Spaces of projective unitary representations}

Let $\mathcal{H}$ be a separable and infinite dimensional Hilbert space. Let $U(\mathcal{H})$ denote the group of unitary operators of $\mathcal{H}$ and let $P U(\mathcal{H})$ be the group of projective unitary operators, defined as the quotient of $U(\mathcal{H})$ by its center,

$$
P U(\mathcal{H})=\frac{U(\mathcal{H})}{\{\zeta \cdot \operatorname{Id}|| \zeta \mid=1\}}=U(\mathcal{H}) / S^{1}
$$

where the center $Z(U(\mathcal{H}))$ is identified with $S^{1}$. Then $P U(\mathcal{H})$ fits in the following short exact sequence of groups

$$
1 \rightarrow S^{1} \hookrightarrow U(\mathcal{H}) \stackrel{\pi}{\rightarrow} \operatorname{PU}(\mathcal{H}) \rightarrow 1 .
$$

and moreover in (Simms, 1970, Thm. 1) it is shown that the above sequence is a $S^{1}$-principal bundle; in other words, the quotient map $U(\mathcal{H}) \stackrel{\pi}{\rightarrow} P U(\mathcal{H})$ has local cross sections.

The group of projective unitary operators may be endowed with the strong operator topology, and in (Simms, 1970, Thm. 1) it is shown that this topology agrees with the quotient topology. Since $S^{1}$ acts on $U(\mathcal{H})$ by isometries, we may endow the group $P U(\mathcal{H})$ with the metric defined by the distance between the orbits, i.e. for $T, U \in P U(\mathcal{H})$ define

$$
\begin{aligned}
& \langle\langle T, U\rangle\rangle:= \\
& \quad \min \left\{\langle\widetilde{T}, \widetilde{U}\rangle: \widetilde{T} \in \pi^{-1}(T), \widetilde{U} \in \pi^{-1}(U)\right\} .
\end{aligned}
$$

Let $G$ be a compact Lie group and let

$$
\alpha: G \rightarrow P U(\mathcal{H})
$$

be a continuous homomorphism. The homomorphism $\alpha$ defines a projective and unitary representation of $G$ on the projective Hilbert space $\mathbb{P} \mathcal{H}:=\mathcal{H}-\{0\} / \mathbb{C}^{*}$.

Every homomorphism $\alpha: G \rightarrow P U(\mathcal{H})$ defines a group $\widetilde{G}_{\alpha}:=\alpha^{*} U(\mathcal{H})$ as the pullback of $U(\mathcal{H})$ under $\alpha$. The group $\widetilde{G}_{\alpha}$ is a $S^{1}$-central extension of the group $G$ and fits into the diagram

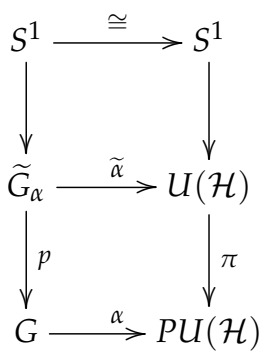

where the bottom square is a pullback square, $\widetilde{\alpha}: \widetilde{G}_{\alpha} \rightarrow$ $U(\mathcal{H})$ is the induced continuous homomorphism and $p: \widetilde{G}_{\alpha} \rightarrow G$ is the projection homomorphism.

10
Since the kernel of the homomorphism

$$
p: \widetilde{G}_{\alpha} \rightarrow G
$$

acts on $\mathcal{H}$ by multiplication, we only need to consider irreducible representations of the group $\widetilde{G}_{\alpha}$ where the kernel of $p$ acts by multiplication. Consider the set

$$
\begin{array}{r}
\mathcal{S}\left(\widetilde{G}_{\alpha}\right):=\left\{V \in \operatorname{Irrep}\left(\widetilde{G}_{\alpha}\right) \mid \operatorname{ker}\left(p: \widetilde{G}_{\alpha} \rightarrow G\right)\right. \\
\text { acts by multiplication on } V\}
\end{array}
$$

and make the following definition:

Definition 5.1. A continuous homomorphism $\alpha$ : $G \rightarrow \operatorname{PU}(\mathcal{H})$ will be called stable whenever the induced homomorphism $\widetilde{\alpha}: \widetilde{G}_{\alpha} \rightarrow U(\mathcal{H})$ belongs to $\operatorname{hom}_{\text {st }}\left(\widetilde{G}_{\alpha}, U(\mathcal{H})\right)_{\mathcal{S}\left(\widetilde{G}_{\alpha}\right)}$. Denote the set of stable homomorphisms from $G$ to $P U(\mathcal{H})$ by $\operatorname{hom}_{\text {st }}(G, P U(\mathcal{H}))$.

Since $G$ is compact and $P U(\mathcal{H})$ is a metric space, the set of stable homomorphisms

$$
\operatorname{hom}_{\text {st }}(G, P U(\mathcal{H}))
$$

may be endowed with the supremum metric, i.e. for $\alpha, \beta \in \operatorname{hom}_{\text {st }}(G, P U(\mathcal{H}))$ let

$$
\langle\langle\alpha, \beta\rangle\rangle:=\sup \{\langle\langle\alpha(g), \beta(g)\rangle\rangle \mid g \in G\} .
$$

By (Munkres, 2000, Thm. 46.8) this metric induces the compact-open topology on $\operatorname{hom}_{\mathrm{st}}(G, P U(\mathcal{H}))$.

Recall that a $S^{1}$-central extension of a Lie group $G$ is an extension $\widetilde{G}$ of $G$ which fits in the short exact sequence of Lie groups

$$
1 \rightarrow S^{1} \rightarrow \widetilde{G} \stackrel{p}{\rightarrow} G \rightarrow 1
$$

and such that $S^{1}$ is a subgroup of the center $Z(\widetilde{G})$. Since Lie groups are locally compact and $S^{1}$ is compact, the projection map $\widetilde{G} \stackrel{p}{\rightarrow} G$ is moreover a $S^{1}$-principal bundle.

Two $S^{1}$-central extensions

$$
\widetilde{G}_{0} \stackrel{p_{0}}{\rightarrow} G \text { and } \widetilde{G}_{1} \stackrel{p_{1}}{\rightarrow} G
$$

of $G$ are isomorphic as $S^{1}$-central extensions, if there exist an isomorphism of groups

$$
\phi: \widetilde{G}_{0} \cong \widetilde{G}_{1}
$$

such that $p_{1} \circ \phi=p_{0}$. Denote by $\operatorname{Ext}\left(G, S^{1}\right)$ the set of isomorphism classes of $S^{1}$-central extensions of $G$ and denote by $[\widetilde{G}]$ the isomorphism class of an extension.

Proposition 5.2. Let $G$ be a compact Lie group. Then the canonical map

$$
\operatorname{hom}_{\mathrm{st}}(G, P U(\mathcal{H})) \rightarrow \operatorname{Ext}\left(G, S^{1}\right), \quad \alpha \mapsto\left[\widetilde{G}_{\alpha}\right]
$$

induces an isomorphism of sets at the level of the conjugacy classes of stable homomorphisms, i.e.

$$
\operatorname{hom}_{\text {st }}(G, P U(\mathcal{H})) / P U(\mathcal{H}) \cong \operatorname{Ext}\left(G, S^{1}\right) .
$$


Proof. Let us show first that every isomorphism class of a $S^{1}$-central extension arises as the pullback of a stable homomorphism from $G$ to $P U(\mathcal{H})$. Consider an extension $\widetilde{G} \rightarrow G$, take the Hilbert space $\mathcal{H}^{\prime}:=L^{2}(\widetilde{G}) \otimes L^{2}([0,1])$ where $L^{2}$ denotes square integrable functions and take the standard action of $\widetilde{G}$ on $L^{2}(\widetilde{G})$. By Peter-Weyl's Theorem the Hilbert space $\mathcal{H}^{\prime}$ contains each irreducible representations of $\widetilde{G}$ infinitely number of times. Take the isotypical part corresponding to the irreducible representations in $\mathcal{S}(\widetilde{G})$

$$
\mathcal{H}:=\bigoplus_{V \in \mathcal{S}(\widetilde{G})} \mathcal{H}^{\prime V}
$$

and note that the induced action of $\widetilde{G}$ on $\mathcal{H}$ is unitary and that the kernel of the projection $\widetilde{G} \rightarrow G$ acts on $\mathcal{H}$ by multiplication. Therefore the action $\widetilde{G} \rightarrow U(\mathcal{H})$ fits into the diagram

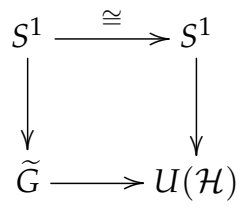

thus inducing a homomorphism $G \rightarrow P U(\mathcal{H})$. Therefore all $S^{1}$-central extensions of $G$ appear as pullbacks of stable homomorphisms from $G$ to $P U(\mathcal{H})$.

Now let us consider two homomorphisms $\alpha, \beta \in$ $\operatorname{hom}_{\text {st }}(G, P U(\mathcal{H}))$ ) which are conjugate, i.e. there exist $F \in P U(\mathcal{H})$ such that $F \alpha(g) F^{-1}=\beta(g)$ for all $g \in G$. Hence the groups $\widetilde{G}_{\alpha}:=\{(g, T) \in G \times U(\mathcal{H}) \mid \alpha(g)=$ $\pi(T)\}$ and $\widetilde{G}_{\beta}:=\{(g, T) \in G \times \mathcal{U}(\mathcal{H}) \mid \beta(g)=\pi(T)\}$ are isomorphic as $S^{1}$-central extensions; the map $\widetilde{G}_{\alpha} \rightarrow \widetilde{G}_{\beta}$, $(g, T) \mapsto\left(g, F T F^{-1}\right)$ is the desired isomorphism. Denoting by $\left.\operatorname{hom}_{\text {st }}(G, P U(\mathcal{H}))\right) / P U(\mathcal{H})$ the set of conjugacy classes of stable homomorphisms we obtain a surjective map

$$
\operatorname{hom}_{\text {st }}(G, P U(\mathcal{H})) / P U(\mathcal{H}) \rightarrow \operatorname{Ext}\left(G, S^{1}\right) .
$$

Let us now suppose that the groups $\widetilde{G}_{\alpha}$ and $\widetilde{G}_{\beta}$ are isomorphic as $S^{1}$-central extensions of $G$. The homomorphisms $\widetilde{\alpha}: \widetilde{G}_{\alpha} \rightarrow U(\mathcal{H})$ and $\widetilde{\beta}: \widetilde{G}_{\beta} \rightarrow U(\mathcal{H})$ induce decompositions of $\mathcal{H}$ by isotypical components $\mathcal{H} \cong \bigoplus_{V \in \mathcal{S}\left(\widetilde{G}_{\alpha}\right)} \mathcal{H}_{\alpha}^{V}$ and $\mathcal{H} \cong \bigoplus_{W \in \mathcal{S}\left(\widetilde{G}_{\beta}\right)} \mathcal{H}_{\beta}^{W}$. The isomorphism $\phi: \widetilde{G}_{\alpha} \cong \widetilde{G}_{\beta}$ induces a canonical isomorphism between the sets $\mathcal{S}\left(\widetilde{G}_{\alpha}\right)$ and $\mathcal{S}\left(\widetilde{G}_{\beta}\right)$, and therefore there exist a unitary isomorphism between $\bigoplus_{V \in \mathcal{S}\left(\widetilde{G}_{\alpha}\right)} \mathcal{H}_{\alpha}^{V}$ and $\bigoplus_{W \in \mathcal{S}\left(\widetilde{G}_{\beta}\right)} \mathcal{H}_{\beta}^{W}$ which is compatible with the actions of the groups and the isomorphism $\phi$. Therefore there exists a unitary isomorphism $F \in U(\mathcal{H})$ such that $F \widetilde{\alpha} F^{-1}=\widetilde{\beta}$ and this implies that $\alpha$ and $\beta$ are conjugate. Hence we have an isomorphism of sets $\operatorname{hom}_{\mathrm{st}}(G, P U(\mathcal{H})) / P U(\mathcal{H}) \stackrel{\cong}{\rightarrow}$ $\operatorname{Ext}\left(G, S^{1}\right)$.

Take representatives $\widetilde{G}$ for each isomorphism class of $S^{1}$ central extension and denote by

$$
\begin{array}{r}
\operatorname{hom}_{\mathrm{st}}(G, P U(\mathcal{H}))_{\widetilde{G}}:=\left\{\alpha \in \operatorname{hom}_{\mathrm{st}}(G, P U(\mathcal{H})) \mid\right. \\
\left.\widetilde{G}_{\alpha} \cong \widetilde{G} \text { as } S^{1} \text {-central extensions of } G\right\}
\end{array}
$$

the space of stable homomorphisms from $G$ to $P U(\mathcal{H})$ which define a $S^{1}$-central extension isomorphic to $\widetilde{G}$. In view of Proposition 5.2 we have that

$$
\begin{aligned}
& \operatorname{hom}_{\text {st }}(G, P U(\mathcal{H}))= \\
& \bigsqcup_{[\widetilde{G}] \in \operatorname{Ext}\left(G, S^{1}\right)} \operatorname{hom}_{\text {st }}(G, P U(\mathcal{H}))_{\widetilde{G}}
\end{aligned}
$$

Consider $\widetilde{\alpha} \in \operatorname{hom}_{\text {st }}(\widetilde{G}, U(\mathcal{H}))_{\mathcal{S}(\widetilde{G})}$ and note that by definition of the set of irreducible representations $\mathcal{S}(\widetilde{G})$, the homomorphism $\widetilde{\alpha}$ makes the following diagram of homomorphisms commutative

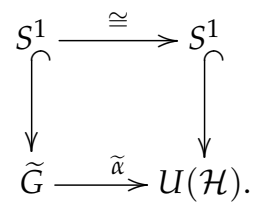

Therefore $\widetilde{\alpha}$ induces a continuous homomorphism $\Psi(\widetilde{\alpha}) \in$ $\operatorname{hom}_{\text {st }}(G, P U(\mathcal{H}))$, and we may define a map of sets

$$
\begin{aligned}
\Psi: \operatorname{hom}_{\text {st }}(\widetilde{G}, U(\mathcal{H}))_{\mathcal{S}(\widetilde{G})} & \rightarrow \operatorname{hom}_{\text {st }}(G, P U(\mathcal{H}))_{\widetilde{G}} \\
\widetilde{\alpha} & \mapsto \Psi(\widetilde{\alpha}) .
\end{aligned}
$$

Now consider the abelian group $\operatorname{hom}\left(G, S^{1}\right)$ of continuous homomorphisms from $G$ to $S^{1}$ endowed with the group structure given by pointwise multiplication. For every

$$
\widetilde{\alpha} \in \operatorname{hom}_{\text {st }}(\widetilde{G}, U(\mathcal{H}))_{\mathcal{S}(\widetilde{G})}
$$

and $\eta \in \operatorname{hom}\left(G, S^{1}\right)$ define $\eta \cdot \widetilde{\alpha}: \widetilde{G} \rightarrow U(\mathcal{H})$ by

$$
\eta \cdot \widetilde{\alpha}(\widetilde{g}):=\eta(\pi(\widetilde{g})) \widetilde{\alpha}(\widetilde{g}) .
$$

The homomorphism $\eta \cdot \widetilde{\alpha}$ belongs to

$$
\operatorname{hom}_{\mathrm{st}}(\widetilde{G}, U(\mathcal{H}))_{\mathcal{S}(\widetilde{G})}
$$

since the action of $\operatorname{ker}(\widetilde{G} \rightarrow G)$ is unaffected and therefore the representations that $\eta \cdot \widetilde{\alpha}$ define belong to $\mathcal{S}(\widetilde{G})$. 
Therefore we have an action of $\operatorname{hom}\left(G, S^{1}\right)$ on $\operatorname{hom}_{\text {st }}(\widetilde{G}, U(\mathcal{H}))_{\mathcal{S}(\widetilde{G})}$ as follows:

$$
\begin{aligned}
& \operatorname{hom}\left(G, S^{1}\right) \times \operatorname{hom}_{\text {st }}(\widetilde{G}, U(\mathcal{H}))_{\mathcal{S}(\widetilde{G})} \rightarrow \\
& \operatorname{hom}_{\text {st }}(\widetilde{G}, U(\mathcal{H}))_{\mathcal{S}(\widetilde{G})} \\
&(\eta, \widetilde{\alpha}) \mapsto \eta \cdot \widetilde{\alpha} .
\end{aligned}
$$

We claim the following theorem.

Theorem 5.3. Let $G$ be a compact Lie group which is connected or abelian, and let $\widetilde{G}$ be a $S^{1}$-central extension of $G$. Let $\mathcal{S}(\widetilde{G})$ be the set of isomorphism classes of irreducible representations of $\widetilde{G}$ on which $\operatorname{ker}(\widetilde{G} \rightarrow G)$ acts by multiplication of scalars. Then the map

$$
\begin{aligned}
\Psi: \operatorname{hom}_{\mathrm{st}}(\widetilde{G}, U(\mathcal{H}))_{\mathcal{S}(\widetilde{G})} & \rightarrow \operatorname{hom}_{\text {st }}(G, P U(\mathcal{H}))_{\widetilde{G}} \\
\widetilde{\alpha} & \mapsto \Psi(\widetilde{\alpha})
\end{aligned}
$$

is a $\operatorname{hom}\left(G, S^{1}\right)$-principal bundle, and in particular a local homeomorphism.

Proof. We already know that $\Psi$ is surjective; the space $\operatorname{hom}_{\text {st }}(G, P U(\mathcal{H}))_{\widetilde{G}}$ consists of the homomorphisms $\alpha$ such that $\widetilde{G}_{\alpha} \cong \widetilde{G}$ as $S^{1}$-principal bundles. The continuity of $\Psi$ follows from the inequality $\langle\widetilde{\alpha}, \widetilde{\beta}\rangle \geq\langle\langle\Psi(\widetilde{\alpha}), \Psi(\widetilde{\beta})\rangle\rangle$ since by definition

$$
\begin{aligned}
\langle\langle\Psi(\widetilde{\alpha}), \Psi(\widetilde{\beta})\rangle\rangle & =\sup _{g \in G}\langle\langle\Psi(\widetilde{\alpha})(g), \Psi(\widetilde{\beta})(g)\rangle\rangle \\
& \leq \sup _{\widetilde{g} \in \widetilde{G}}\langle\widetilde{\alpha}(\widetilde{g}), \widetilde{\beta}(\widetilde{g})\rangle=\langle\widetilde{\alpha}, \widetilde{\beta}\rangle .
\end{aligned}
$$

Take now $\widetilde{\alpha}, \widetilde{\alpha}^{\prime}$ such that $\Psi(\widetilde{\alpha})=\Psi\left(\widetilde{\alpha}^{\prime}\right)$. Note that for all $\widetilde{g} \in \widetilde{G}$ the product $\widetilde{\alpha}(\widetilde{g}) \widetilde{\alpha}^{\prime}(\widetilde{g})^{-1}$ belongs to $S^{1} \subset U(\mathcal{H})$ and therefore we can define the assignment

$$
\widetilde{\eta}: \widetilde{G} \rightarrow S^{1}, \quad \widetilde{g} \mapsto \widetilde{\alpha}(\widetilde{g}) \widetilde{\alpha}^{\prime}(\widetilde{g})^{-1} .
$$

This assignment is indeed a homomorphism since we have the equalities

$$
\begin{aligned}
\widetilde{\eta}(\widetilde{g} \widetilde{h}) & =\widetilde{\alpha}(\widetilde{g} \widetilde{h}) \widetilde{\alpha}^{\prime}(\widetilde{g} \widetilde{h})^{-1} \\
& =\widetilde{\alpha}(\widetilde{g}) \widetilde{\alpha}(\widetilde{h}) \widetilde{\alpha}^{\prime}(\widetilde{h})^{-1} \widetilde{\alpha}^{\prime}(\widetilde{g})^{-1} \\
& =\widetilde{\alpha}(\widetilde{g}) \widetilde{\eta}(\widetilde{h}) \widetilde{\alpha}^{\prime}(\widetilde{g})^{-1} \\
& =\widetilde{\eta}(\widetilde{g}) \widetilde{\eta}(\widetilde{h})
\end{aligned}
$$

which follow from the fact that $\widetilde{\eta}(\widetilde{h})$ lies on the center of $U(\mathcal{H})$. The homomorphism $\widetilde{\eta}$ is trivial once restricted to $\operatorname{ker}(\widetilde{G} \rightarrow G)$ and therefore it induces a homomorphism $\eta: G \rightarrow S^{1}$ such that $\widetilde{\eta}(\widetilde{g})=\eta(\pi(\widetilde{g}))$. Therefore we obtain the equation $\eta \cdot \widetilde{\alpha}^{\prime}=\widetilde{\alpha}$ which implies that $\Psi$ induces a bijective map at the level of sets

$$
\begin{aligned}
\operatorname{hom}_{\mathrm{st}}(\widetilde{G}, U(\mathcal{H}))_{\mathcal{S}(\widetilde{G})} / \operatorname{hom}\left(G, S^{1}\right) \stackrel{\cong}{\rightrightarrows} \\
\operatorname{hom}_{\mathrm{st}}(G, P U(\mathcal{H}))_{\widetilde{G}} .
\end{aligned}
$$

We need to show now that $\Psi$ is a local homeomorphism. Note that for any non trivial $\eta \in \operatorname{hom}\left(G, S^{1}\right)$ and $\mathbf{1}$ the trivial homomorphism, we have that $\langle\mathbf{1}, \eta\rangle \geq \sqrt{2}$ since any non trivial homomorphism must take at least one value in the subset $\left\{e^{i t} \mid \frac{\pi}{2}<t<\frac{3 \pi}{2}\right\} \subset S^{1}$. This implies that for any $\widetilde{\alpha} \in \operatorname{hom}_{\text {st }}(\widetilde{G}, U(\mathcal{H}))_{\mathcal{S}(\widetilde{G})}$ and any $\eta \in \operatorname{hom}\left(G, S^{1}\right)$ we have

$$
\langle\widetilde{\alpha}, \eta \cdot \widetilde{\alpha}\rangle=\langle\mathbf{1}, \eta\rangle \geq \sqrt{2} ;
$$

therefore if we denote by

$$
B_{\delta}(\widetilde{\alpha}):=\left\{\widetilde{\beta} \in \operatorname{hom}_{\mathrm{st}}(\widetilde{G}, U(\mathcal{H}))_{\mathcal{S}(\widetilde{G})} \mid\langle\widetilde{\alpha}, \widetilde{\beta}\rangle<\delta\right\}
$$

we have that for all $\eta \in \operatorname{hom}\left(G, S^{1}\right)$ with $\eta \neq 1$, the intersection $B_{\delta}(\widetilde{\alpha}) \cap\left(\eta \cdot B_{\delta}(\widetilde{\alpha})\right)=\varnothing$ for $\delta<\frac{1}{2}$, which in particular says that the action of $\operatorname{hom}\left(G, S^{1}\right)$ is completely discontinuous.

Let us restrict the map $\Psi$ to the open set $B_{\delta}(\widetilde{\alpha})$ with $\delta \ll \frac{1}{2}$. By equation (3) we have that the map

$$
\left.\Psi\right|_{B_{\delta}(\widetilde{\alpha})}: B_{\delta}(\widetilde{\alpha}) \rightarrow \Psi\left(B_{\delta}(\widetilde{\alpha})\right)
$$

is bijective and continuous, we claim furthermore that it is a homeomorphism. Let us show that $\left.\Psi\right|_{B_{\delta}(\widetilde{\alpha})}{ }^{-1}$ is continuous.

Let $\widetilde{K}$ be a maximal abelian subgroup of $\widetilde{G}$ and denote by $\widetilde{\alpha}^{\prime}: \widetilde{K} \rightarrow U(\mathcal{H})$ the restriction $\left.\widetilde{\alpha}\right|_{\widetilde{K}}$. Denote by $K \subset G$ the abelian subgroup that $\widetilde{K}$ defines and note that $\widetilde{K} \stackrel{p}{\rightarrow} K$ defines a $S^{1}$-central extension. Denote by

$$
\mathcal{H} \cong \bigoplus_{W \in \mathcal{S}(\widetilde{K})} \mathcal{H}_{\widetilde{\alpha}^{\prime}}^{W}
$$

the decomposition of $\mathcal{H}$ into isotypical components and note that $S^{1}$ acts by multiplication on the one dimensional irreducible representations $W$ of $\mathcal{S}(\widetilde{K})$. Fix $V \in \mathcal{S}(\widetilde{K})$ and choose any unitary vector $x \in \mathcal{H}_{\widetilde{\alpha}^{\prime}}^{V}$.

Take $\varepsilon \in \mathbb{R}$ such that $0<\varepsilon \ll 1$ and choose $\delta<\frac{1}{2}$ such that for any $\widetilde{\beta} \in B_{\delta}(\widetilde{\alpha})$ we have that for all $\widetilde{g} \in \widetilde{G}$

$$
|\widetilde{\alpha}(\widetilde{g}) x-\widetilde{\beta}(\widetilde{g}) x|<\varepsilon ;
$$

this $\delta$ exists by the definition of the strong operator topology and the metric defined in equation (1).

Take a sequence $\left\{\widetilde{\beta}_{n}\right\}_{n \in \mathbb{N}}$ of homomorphisms in $B_{\delta}(\widetilde{\alpha})$ and denote by

$$
\alpha, \beta_{n} \in \operatorname{hom}_{\mathrm{st}}(G, P U(\mathcal{H}))_{\widetilde{G}}
$$


the projective homomorphisms that $\widetilde{\alpha}$ and $\widetilde{\beta}_{n}$ define. Assume that $\lim _{n \rightarrow \infty} \beta_{n}=\alpha$; let us show that this implies that $\lim _{n \rightarrow \infty} \widetilde{\beta}_{n}=\widetilde{\alpha}$.

Since we have that $\lim _{n \rightarrow \infty} \beta_{n}=\alpha$, there must exist unitary complex numbers $\lambda_{n}(g) \in S^{1}$ such that for all $\widetilde{g} \in \widetilde{G}$

$$
\lim _{n \rightarrow \infty} \lambda_{n}(g) \widetilde{\beta}_{n}(\widetilde{g})=\widetilde{\alpha}(\widetilde{g})
$$

Take $\sigma \in \mathbb{R}$ such that $0<\sigma \ll 1$ and let $N \in \mathbb{N}$ be such that for all $n>N$ and all $\widetilde{g} \in \widetilde{G}$ we have

$$
\left|\lambda_{n}(g) \widetilde{\beta}_{n}(\widetilde{g}) x-\widetilde{\alpha}(\widetilde{g}) x\right|<\sigma .
$$

Denote by $\chi_{W}: \widetilde{K} \rightarrow S^{1}$ the characters of the irreducible representations $W$ of the abelian group $\widetilde{K}$, and write $x=\sum_{W \in \mathcal{S}(\widetilde{K})} y_{n}^{W}$ in terms of the decomposition on isotypical components $\mathcal{H} \cong \bigoplus_{W \in \mathcal{S}(\widetilde{K})} \mathcal{H}_{\widetilde{\beta}_{n}^{\prime}}^{W}$ relative to $\widetilde{\beta}_{n}^{\prime}$ with $y_{n}^{W} \in \mathcal{H}_{\widetilde{\beta}_{n}^{\prime}}^{W}$. We obtain that for all $\widetilde{k} \in \widetilde{K}$

$$
\widetilde{\alpha}(\widetilde{k}) x=\chi_{V}(\widetilde{k}) x=\sum_{W} \chi_{V}(\widetilde{k}) y_{n}^{W}
$$

and

$$
\widetilde{\beta}_{n}(\widetilde{k}) x=\sum_{W} \chi_{W}(\widetilde{k}) y_{n}^{W},
$$

and therefore by equation (4)

$$
\left|\widetilde{\beta}_{n}(\widetilde{k}) x-\widetilde{\alpha}(\widetilde{k}) x\right|=\left|\sum_{W \neq V}\left(\chi_{W}(\widetilde{k})-\chi_{V}(\widetilde{k})\right) y_{n}^{W}\right|<\varepsilon
$$

and by equation (5)

$$
\begin{aligned}
&\left|\lambda_{n}(k) \widetilde{\beta}_{n}(\widetilde{k}) x-\widetilde{\alpha}(\widetilde{k}) x\right|= \\
&\left|\chi_{V}(\widetilde{k})\left(\lambda_{n}(k)-1\right) y_{n}^{V}+\sum_{W \neq V}\left(\chi_{W}(\widetilde{k})-\chi_{V}(\widetilde{k})\right) y_{n}^{W}\right| \\
&<\sigma .
\end{aligned}
$$

Since for all $\widetilde{k} \in \widetilde{K}$ we have that

$$
\left|\sum_{W \neq V}\left(\chi_{W}(\widetilde{k})-\chi_{V}(\widetilde{k})\right) y_{n}^{W}\right|<\varepsilon,
$$

Lemma 5.5 shows that $\left|\sum_{W \neq V} y_{n}^{W}\right|<\varepsilon$. Therefore we obtain

$$
\left|y_{n}^{V}\right|=\left|x-\sum_{W \neq V} y_{n}^{W}\right|>1-\varepsilon
$$

Since the vectors $y_{n}^{W}$ are pairwise orthogonal, the inequality (6) implies that

$$
\left|\chi_{V}(\widetilde{k})\left(\lambda_{n}(k)-1\right) y_{n}^{V}\right|=\left|\lambda_{n}(k)-1\right|\left|y_{n}^{V}\right|<\sigma
$$

and since $\left|y_{n}^{V}\right|>1-\varepsilon$, we have that for all $k \in K$ and all $n>N$

$$
\left|\lambda_{n}(k)-1\right|<\frac{\sigma}{1-\varepsilon}
$$

Since $\varepsilon$ is fixed, we conclude that for all $k \in K$, $\lim _{n \rightarrow \infty} \lambda_{n}(k)=1$. Hence for all $\widetilde{k} \in \widetilde{K}$

$$
\lim _{n \rightarrow \infty} \widetilde{\beta}_{n}(\widetilde{k})=\lim _{n \rightarrow \infty} \lambda_{n}(k) \widetilde{\beta}_{n}(\widetilde{k})=\widetilde{\alpha}(\widetilde{k})
$$

and therefore $\lim _{n \rightarrow \infty} \widetilde{\beta}_{n}^{\prime}=\widetilde{\alpha}^{\prime}$. Now, the restriction map

$$
\begin{aligned}
\operatorname{hom}_{\text {st }}(\widetilde{G}, U(\mathcal{H}))_{\mathcal{S}(\widetilde{G})} & \rightarrow \operatorname{hom}_{\text {st }}(\widetilde{K}, U(\mathcal{H}))_{\mathcal{S}(\widetilde{K})} \\
\widetilde{\alpha} & \mapsto \widetilde{\alpha}^{\prime}=\left.\widetilde{\alpha}\right|_{\widetilde{K}}
\end{aligned}
$$

is an embedding since $\widetilde{G}$ is connected and any representation of $\widetilde{G}$ is uniquely determined by its restriction to $\widetilde{K}$; hence we conclude that

$$
\lim _{n \rightarrow \infty} \widetilde{\beta}_{n}=\widetilde{\alpha}
$$

Therefore $\left.\Psi\right|_{B_{\delta}(\widetilde{\alpha})}: B_{\delta}(\widetilde{\alpha}) \rightarrow \Psi\left(B_{\delta}(\widetilde{\alpha})\right)$ is a homeomorphism and the theorem follows.

Corollary 5.4. Let $G$ be a compact Lie group which is connected or abelian, and let $\widetilde{G}$ be a $S^{1}$-central extension of $G$. Then $\operatorname{hom}_{\mathrm{st}}(G, P U(\mathcal{H}))_{\widetilde{G}}$ is a $K\left(\operatorname{hom}\left(G, S^{1}\right), 1\right)$, namely it is connected, its fundamental group is $\operatorname{hom}\left(G, S^{1}\right)$ and its higher homotopy groups are trivial.

Proof. The result follows from Theorem 5.3 where it is proven that

$$
\operatorname{hom}_{\text {st }}(\widetilde{G}, U(\mathcal{H}))_{\mathcal{S}(\widetilde{G})} \rightarrow \operatorname{hom}_{\text {st }}(G, P U(\mathcal{H}))_{\widetilde{G}}
$$

is a $\operatorname{hom}\left(G, S^{1}\right)$-principal bundle, and from Theorem 4.11 where it is proven that

$$
\operatorname{hom}_{\mathrm{st}}(\widetilde{G}, U(\mathcal{H}))_{\mathcal{S}(\widetilde{G})}
$$

is weakly homotopy equivalent to a point.

Lemma 5.5. Let $\widetilde{K}$ be an abelian compact Lie group and denote by $\chi_{W}: \widetilde{K} \rightarrow S^{1}$ the character of the 1-dimensional irreducible representation $W$. For each irreducible representation $W$ take a vector $y^{W} \in \mathcal{H}$ and assume that $y^{W} \perp y^{Z}$ for $W \neq Z$. Suppose that for all $\widetilde{k} \in \widetilde{K}$

$$
\left|\sum_{W \neq V}\left(\chi_{W}(\widetilde{k})-\chi_{V}(\widetilde{k})\right) y_{n}^{W}\right|<\varepsilon,
$$

then $\left|\sum_{W \neq V} y_{n}^{W}\right|<\varepsilon$. 
Proof. Note first that $\chi_{W}\left(\chi_{V}\right)^{-1}=\chi_{W \otimes V^{-1}}$ and therefore

$$
\begin{aligned}
\left|\sum_{W \neq V}\left(\chi_{W}(\widetilde{k})-\chi_{V}(\widetilde{k})\right) y^{W}\right| & = \\
& \left|\sum_{Z \neq \mathbf{1}}\left(\chi_{Z}(\widetilde{k})-1\right) y^{V \otimes Z}\right| ;
\end{aligned}
$$

taking $x^{Z}:=y^{V \otimes Z}$ we have that for all $\widetilde{k} \in \widetilde{K}$

$$
\left|\sum_{Z \neq \mathbf{1}}\left(\chi_{Z}(\widetilde{k})-1\right) x^{Z}\right|<\varepsilon .
$$

Since $\widetilde{K}$ is isomorphic to a product of cyclic groups, we claim that it is enough to show the lemma whenever $\widetilde{K}$ is $S^{1}$.

Here the irreducible representations of $S^{1}$ are parametrized by $n \in \mathbb{Z}$ and our hypothesis becomes

$$
\left|\sum_{n \neq 0}\left(e^{2 \pi i n j}-1\right) x_{n}\right|<\varepsilon
$$

for all $j \in \mathbb{R}$. Take a prime number $p \in \mathbb{N}$ and consider the inequality for $j=\frac{1}{2 p}, \frac{2}{2 p}, \ldots, \frac{2 p-1}{2 p}$; by the triangle inequality we have

$$
\begin{aligned}
\left|\sum_{r=1}^{2 p-1} \sum_{n \neq 0}\left(e^{2 \pi i n \frac{r}{2 p}}-1\right) x_{n}\right| & \leq \sum_{r=1}^{2 p-1}\left|\sum_{n \neq 0}\left(e^{2 \pi i n \frac{r}{2 p}}-1\right) x_{n}\right| \\
& <(2 p-1) \varepsilon
\end{aligned}
$$

which we may reorder thus obtaining

$$
\begin{aligned}
(2 p-1) \varepsilon & >\left|\sum_{r=1}^{2 p-1} \sum_{n \neq 0}\left(e^{2 \pi i n \frac{r}{2 p}}-1\right) x_{n}\right| \\
& =\left|\left(\sum_{n: p \nmid n} x_{n}\right)\left(\sum_{r=1}^{2 p-1}\left(e^{2 \pi i n \frac{r}{2 p}}-1\right)\right)\right| \\
& =2 p\left|\sum_{n: p \nmid n} x_{n}\right|
\end{aligned}
$$

since $\sum_{r=1}^{2 p-1}\left(e^{2 \pi i n \frac{r}{2 p}}-1\right)=-2 p$ whenever $p \nmid n$.

Therefore we have that for all prime $p$ we have that the inequalities

$$
\left|\sum_{n: p \nmid n} x_{n}\right|<\frac{(2 p-1) \varepsilon}{2 p}<\varepsilon
$$

hold, implying the desired result, namely that

$$
\left|\sum_{n \neq 0} x_{n}\right|<\varepsilon \text {. }
$$

The iteration of the previous argument shows the lemma for any abelian compact Lie group.
Theorem 5.6. Let $G$ be a compact Lie group which is connected or abelian, and let $\widetilde{G}$ be a $S^{1}$-central extension of $G, B$ a connected paracompact space and $f: B \rightarrow \operatorname{hom}_{\mathrm{st}}(G, P U(\mathcal{H}))_{\widetilde{G}} a$ continuous map. Then for all $b_{0} \in B$ there exist a neighborhood $V \subset B$ of $b_{0} \in V$ such that $\left.f\right|_{V}: V \rightarrow \operatorname{hom}_{\text {st }}(G, P U(\mathcal{H}))_{\widetilde{G}}$ has an extension $\sigma_{V}: V \rightarrow P U(\mathcal{H})$ that makes the following diagram commutative

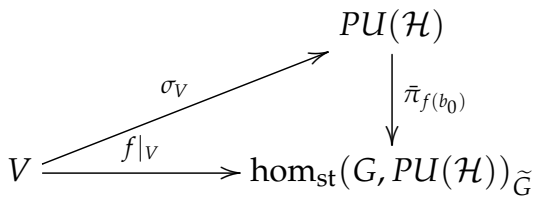

where $\bar{\pi}_{f\left(b_{0}\right)}: \operatorname{PU}(\mathcal{H}) \rightarrow \operatorname{hom}_{\mathrm{st}}(G, P U(\mathcal{H}))_{\widetilde{G}^{\prime}} F \mapsto$ $F f\left(b_{0}\right) F^{-1}$. In particular $f^{*} \mathrm{PU}(\mathcal{H})$ is a $\operatorname{PU}(\mathcal{H})_{f\left(b_{0}\right)^{-}}$ principal bundle where $\operatorname{PU}(\mathcal{H})_{f\left(b_{0}\right)}:=\{F \in \operatorname{PU}(\mathcal{H}) \mid$ $\left.F f\left(b_{0}\right) F^{-1}=f\left(b_{0}\right)\right\}$.

Proof. Take any lift $\widetilde{\alpha} \in \operatorname{hom}_{\text {st }}(\widetilde{G}, U(\mathcal{H}))_{\mathcal{S}(\widetilde{G})}$ such that $\Psi(\widetilde{\alpha})=f\left(b_{0}\right)$ and consider the following commutative diagram

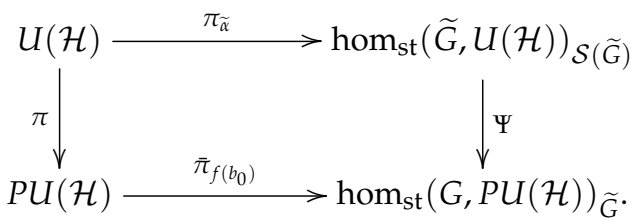

By Theorem 5.3 there exists a neighborhood $W$ of $\widetilde{\alpha}$ such that $\left.\Psi\right|_{W}: W \rightarrow \Psi(W)$ is a homeomorphism. Let $V:=f^{-1}(\Psi(W))$ and consider the map $\tilde{f}: V \rightarrow W$, $b \mapsto\left(\left.\Psi\right|_{V}\right)^{-1}(f(b))$. By Theorem 4.9 and Corollary 4.10 there exist a section $s: V \rightarrow U(\mathcal{H})$ such that $s(b) \widetilde{\alpha} s(b)^{-1}=\widetilde{f}(b)$. The composition $\sigma_{V}: V \rightarrow P U(\mathcal{H})$, $\sigma_{V}:=\pi \circ s$ is desired local extension of $f$ since we have

$$
\begin{aligned}
\sigma_{V}(b) f\left(b_{0}\right) \sigma_{V}(b)^{-1} & =\pi\left(s(b) \widetilde{\alpha} s(b)^{-1}\right) \\
& =\pi(\widetilde{f}(b))=f(b) .
\end{aligned}
$$

The map $V \times\left. P U(\mathcal{H})_{f\left(b_{0}\right)} \rightarrow\left(f^{*} \operatorname{PU}(\mathcal{H})\right)\right|_{V},(b, F) \mapsto$ $\left(b, \sigma_{V}(b) F\right)$ is the desired local trivialization for the $\operatorname{PU}(\mathcal{H})_{f\left(b_{0}\right)}$-principal bundle

$$
\begin{aligned}
& f^{*} \operatorname{PU}(\mathcal{H}):= \\
& \quad\left\{(b, F) \in B \times P U(\mathcal{H}) \mid f(b)=\bar{\pi}_{f\left(b_{0}\right)}(F)\right\} .
\end{aligned}
$$




\section{Applications and further research}

One very important application of the existence of lifts for paracompact spaces shown in Theorem 5.6, is the construction of universal equivariant projective unitary and stable bundles necessary for the study of the twisted equivariant K-theory as an equivariant parametrized cohomology theory (see (Barcenas, Espinoza, Joachim and Uribe, 2014, Chapter 5) and (Atiyah and Segal, 2004, Chapter 6)). The construction of these universal bundles relies on the construction of classifying spaces for certain families for subgroups, together with explicit topological properties that the groups and the associated spaces of homomorphisms need to satisfy. In what follows we will review these constructions and we will show the implications that the results proved in the previous sections have on the existence of universal equivariant projective unitary bundles.

Let $G$ and $P$ be topological groups. A G-equivariant $P$-principal bundle consists of a $P$-principal bundle $p$ : $E \rightarrow X$ together with left $G$ actions on $E$ and $X$ commuting with the right $P$ action on $E$ such that $p$ is $G$ equivariant. For every $e \in E$ we obtain a local representation $\rho_{e}: G_{p(e)} \rightarrow P$ determined by $g^{-1} \cdot e=e \cdot \rho_{e}(g)$ for $g \in G_{p(e)}$ where $G_{p(e)}$ is the isotropy group of $p(e) \in X$.

In (Lück and Uribe, 2014, Thm. 11.4) it was constructed a universal G-equivariant $P$-principal bundle with a prescribed family of local representations through the use of classifying spaces of families of subgroups. The fact that these classifying spaces of families of subgroups permitted to obtain equivariant principal bundles relied on topological properties of the groups $G$ and $P$ and on the spaces of prescribed homomorphisms. Let us recall the main ingredients.

A family $\mathcal{R}$ of local representations for $(G, P)$ is a set of pairs $(H, \alpha)$, where $H$ is a subgroup of $G$ and $\alpha: H \rightarrow P$ is a continuous group homomorphism, such that the family is closed under finite intersections, under conjugation in $P$ and under conjugation in $G$ (see (Lück and Uribe, 2014, Def. 3.3) for a detailed description).

It is said (Lück and Uribe, 2014, Def. 6.1) that the family $\mathcal{R}$ satisfies Condition $(\mathrm{H})$ if the following holds for every $(H, \alpha) \in \mathcal{R}$ :

- The path component of $\alpha$ in $\operatorname{hom}(H, P)$ is contained in the orbit $\left\{p \alpha p^{-1} \mid p \in P\right\}$.

- The projection $P \rightarrow P / P_{\alpha}$ has a local cross section where $P_{\alpha}=\left\{p \in P \mid p \alpha p^{-1}=\alpha\right\}$ is the isotropy group of $\alpha$ under the conjugation action of $P$.
- The projection $G \rightarrow G / H$ has a local cross section.

- The canonical map

$$
\begin{aligned}
\iota_{\alpha}: P / P_{\alpha} & \rightarrow \operatorname{hom}(H, P) \\
p P_{\alpha} & \mapsto p \alpha p^{-1}
\end{aligned}
$$

is a homeomorphism into its image.

To a family of local representations $\mathcal{R}$ we can associate a family of subgroups of $G \times P$ consisting of the set $\mathcal{F}(\mathcal{R}):=\{K(H, \alpha) \mid(H, \alpha) \in \mathcal{R}\}$ where $K(H, \alpha):=$ $\{(g, \alpha(g)) \mid g \in H\}$. Let

$$
E(G, P, \mathcal{R}):=E_{\mathcal{F}(\mathcal{R})}(G \times P)
$$

be the classifying space for the family of subgrups $\mathcal{F}(\mathcal{R})$, i.e. a $(G \times P)$-CW-complex whose isotropy groups belong to $\mathcal{F}(\mathcal{R})$ and for which the $K(H, \alpha)$-fixed point set $E_{\mathcal{F}(\mathcal{R})}(G \times P)^{K(H, \alpha)}$ is nonempty and weakly contractible for every $(H, \alpha) \in \mathcal{R}$.

Theorem 11.4 of Lück and Uribe (2014) claims that if the family of local representations $\mathcal{R}$ satisfies Condition $(\mathrm{H})$, then $E(G, P, \mathcal{R}) \rightarrow E(G, P, \mathcal{R}) / P$ is a $G$-equivariant $P$-principal bundle which is moreover universal for $G$ equivariant $P$-principal bundles whose local representations appear in $\mathcal{R}$.

In this paper we are interested in $G$ equivariant projective unitary stable bundles, namely G-equivariant $P U(\mathcal{H})$ principal bundles whose local representations $(H, \alpha)$ consist of stable homomorphisms $\alpha: H \rightarrow P U(\mathcal{H})$ as were defined in Definition 5.1.

Whenever $P U(\mathcal{H})$ is endowed with the norm topology (let us denote it by $\left.\operatorname{PU}(\mathcal{H})_{n}\right), G$ is a topological group and $\mathcal{S}$ consists of the family of local representations $(H, \alpha)$ where $H$ is a finite subgroup of $G$ and $\alpha \in \operatorname{hom}_{\text {st }}\left(H, P U(\mathcal{H})_{n}\right)$ is a stable homomorphism. Theorem 15.12 of Lück and Uribe (2014) shows that the bundle

$$
E\left(G, P U(\mathcal{H})_{n}, \mathcal{S}\right) \rightarrow E\left(G, P U(\mathcal{H})_{n}, \mathcal{S}\right) / P U(\mathcal{H})_{n}
$$

is a universal $G$ equivariant projective unitary stable bundle for almost free G-CW-complexes

It would be expected that a similar statement would hold whenever we expand the family of local representations for pairs $(H, \alpha)$ where $H$ is a compact Lie group and $\alpha$ is a stable homomorphism. Unfortunately this is not the case for the following reasons: whenever $H$ is a compact Lie group which is not finite, the space of stable homomorphisms to $\operatorname{PU}(\mathcal{H})_{n}$ in the norm topology is empty, i.e. $\operatorname{hom}_{\text {st }}\left(H, P U(\mathcal{H})_{n}\right)=\varnothing$. 
If we consider the group $P U(\mathcal{H})$ endowed with the strong operator topology, as it is done throughout this article, the family of local representations $\mathcal{S}$ consisting of pairs $(H, \alpha)$ with $H$ a compact Lie group and $\alpha \in \operatorname{hom}_{\text {st }}(H, P U(\mathcal{H}))$ does not satisfy Condition $(\mathrm{H})$ of Theorem 11.4 of Lück and Uribe (2014). In particular the canonical map

$$
\operatorname{PU}(\mathcal{H}) / P U(\mathcal{H})_{\alpha} \rightarrow \operatorname{hom}_{\text {st }}(H, P U(\mathcal{H}))
$$

is not a homeomorphism into its image, since Theorem 4.8 implies that canonical map

$$
P U(\mathcal{H}) \rightarrow \operatorname{hom}_{\mathrm{st}}(H, P U(\mathcal{H})), \quad F \rightarrow F \alpha F^{-1}
$$

has no local sections.

Nevertheless, by Theorem 5.6 we know that local lifts exists if we restrict to maps

$$
B \rightarrow \operatorname{hom}_{\mathrm{st}}(H, P U(\mathcal{H}))
$$

with $B$ paracompact. Hence we might say that a Weak Condition $(\mathrm{H})$ is satisfied whenever Condition $(\mathrm{H})$ holds on the image of maps $B \rightarrow \operatorname{hom}_{\mathrm{st}}(H, P U(\mathcal{H}))$ where $B$ is paracompact. With this setup in mind, we conjecture that the space

$$
E(G, P U(\mathcal{H}), \mathcal{S}) / P U(\mathcal{H})
$$

would become a universal space for G-equivariant projective unitary stable bundles whenever we restrict our study to the category of paracompact spaces with proper $G$ actions. If this were the case, we would have a space that would allow us to show that the twisted equivariant K-theory is indeed an equivariant parametrized cohomology theory as defined in May (1996).

Finally note that in order for the previous statement to be true we would need to be able to generalize Theorem 5.6 for compact Lie groups which are not necessarily connected, and we would need to show that the proof of Theorem 11.4 of Lück and Uribe (2014) would work if we restrict only to the image of paracompact spaces. These tasks are beyond the scope of this article and we leave them for further research.

\section{References}

Atiyah, M. and Segal, G. (2004) Twisted K-theory. Ukranian Mathematical Bulletin, 1(3):291-334.

Bárcenas, N., Espinoza, J., Joachim, M. and Uribe,B (2014) Universal twist in equivariant $K$-theory for proper and discrete actions. Proc. Lond. Math. Soc. (3), 108(5):1313-1350.

Dixmier, J. and Douady, A. (1963). Champs continus d'espaces hilbertiens et de $C^{*}$-algèbres. Bull. Soc. Math. France, 91:227-284.

Espinoza, J. and Uribe, B. (2014). Topological properties of the unitary group. JP Journal of Geometry and Topology, 16(1):45-55.

Jänich, K. (1965). Vektorraumbündel und der Raum der Fredholm-Operatoren. Math. Ann., 161:129-142.

Lück, W. and Uribe, B. (2014). Equivariant principal bundles and their classifying spaces. Algebraic and Geometric Topology, 14(4):1925-1995.

May, J. P. (1996). Equivariant homotopy and cohomology theory, volume 91 of CBMS Regional Conference Series in Mathematics. Published for the Conference Board of the Mathematical Sciences, Washington, DC; by the American Mathematical Society, Providence, RI.

May, J. P. and Sigurdsson, J. (2006). Parametrized homotopy theory, volume 132 of Mathematical Surveys and Monographs. American Mathematical Society, Providence, RI.

Munkres, J. R. (2000). Topology, Second Edition. PrenticeHall, Inc., Englewood Cliffs, N.J.

Simms, D. J. (1970) Topological aspects of the projective unitary group. Proc. Camb. Phil. Soc., 68:57-60. 